2019-02-01

\title{
Wave radiation from a truncated cylinder of arbitrary cross section
}

Yu, $\mathrm{H}$

http://hdl.handle.net/10026.1/13738

10.1016/j.oceaneng.2019.01.013

Ocean Engineering

Elsevier

All content in PEARL is protected by copyright law. Author manuscripts are made available in accordance with publisher policies. Please cite only the published version using the details provided on the item record or document. In the absence of an open licence (e.g. Creative Commons), permissions for further reuse of content should be sought from the publisher or author. 
1 Title:

2 Wave radiation from a truncated cylinder of arbitrary cross section

4 Author names and affiliations:

$5 \quad$ Huifeng $\mathrm{Yu}^{\mathrm{a}}$, Siming Zheng ${ }^{\mathrm{a}, \mathrm{b}}$, Yongliang Zhanga ${ }^{\mathrm{a}}$, Gregorio Iglesias ${ }^{\mathrm{c}, \mathrm{b}}$

6

7 a State Key Laboratory of Hydroscience and Engineering, Tsinghua University, Beijing 8 100084, China

9 b School of Engineering, University of Plymouth, Drake Circus, Plymouth PL4 8AA, UK

10 c MaREI, Environmental Research Institute \& School of Engineering, University College

11 Cork, Western Rd., Cork, Ireland

12

13

14

15

16

17

18

19

20

21

22

23
Corresponding author: Siming Zheng

Tel: +8601062785659

E-mail address: siming.zheng@plymouth.ac.uk

Received 5 February 2018

Revised 17 September 2018

Accepted 4 January 2019

Available online 18 January 2019

https://doi.org/10.1016/j.oceaneng.2019.01.013 
Abstract: In this paper, a semi-analytical model for solving wave radiation from a truncated cylinder of arbitrary cross section is presented based on linear potential flow theory. The water domain is divided into the interior domain beneath the cylinder and the exterior domain outside the vertical cylinder column. Radiated spatial potentials in these subdomains are expressed as a series expansion of eigen-functions using the method of separation of variables. The continuity conditions for pressure and velocity are satisfied at the interface of the two domains, where the Fourier series expansion method is employed to deal with the radius function associated terms. Therefore, the unknown coefficients in the radiated potential expressions are determined by means of the eigen-function matching method. Hydrodynamic coefficients of the truncated cylinder are evaluated directly based on the radiated spatial potentials. Case studies on wave radiation from a truncated cylinder with "cosine" and "circular" cross sections show a good agreement between the semi-analytical results of added-mass/radiation damping and numerical ones/published data. The validated semi-analytical model is then adopted to study the hydrodynamic characteristics of truncated cylinders with "circular", "cosine", "elliptical" and "quasi-elliptical" sections. For the latter case, the influence of draft on wave radiation is also investigated.

Keywords: Potential flow; Semi-analytical model; Added-mass; Radiation damping; Truncated cylinder; Wave-structure interaction

\section{Introduction}

The prediction of hydrodynamic forces is important for both researchers and engineers for the design and optimization of offshore marine structures. Indeed, the radiation forces acting on a structure, which are due to the wave radiated as a result of the structure's oscillation, are the object of considerable research interest.

In ocean engineering, truncated circular cylinders have been widely used in many marine structures, such as wave energy converters and Spar platforms (Sudhakar and Nallayarasu, 2011; Zheng and Zhang, 2018). To attenuate waves, large, bluff, ship-like bodies whose cross-section closely resembles an ellipse turn out to be one of the options of floating breakwaters (Williams and Darwiche, 1988). Vertical cylinders with elliptical cross sections can be adopted as the foundations of some coastal/offshore bridges as well (Wan et al., 2017). A more common shape of the bridge's foundation, however, might be a quasi-elliptical section (Liu et al., 2017; Eidem 2017). For all these marine structures, the radiation problem of a cylinder with different cross-sections is involved in either their operation or installation.

As early as 1980s, Yeung (1981) proposed an analytical model to study wave radiation by a truncated circular cylinder floating in finite-depth water. Bessel and modified Bessel functions were used in his model to describe the spatial velocity potential in the water domain in a polar coordinate system. Later, Bhatta and Rahman (2003) and Bhatta (2007) studied both wave diffraction and radiation problems from a truncated cylinder with an analytical method. It was revealed that a larger heave added-mass could be obtained for the cylinder with a larger draft. For the same draft of the cylinder, added mass was found to be larger for smaller water depths. Analytical studies on wave radiation from a vertical circular cylinder in other situations, e.g. in a channel, floating in shallow water, submerged in finite water depth, floating in front of a vertical 
wall, were also carried out (Linton and Evans, 1992; Drobyshevski, 2004; Jiang, et al., 2013; Zheng and Zhang, 2016). The hydrodynamic analysis of arrays of circular cylinders has received attention as well, the brief review of which was given in our previous work (Zheng and Zhang, 2015; 2016; 2018).

As an expansion for the investigation of circular cylinder, wave radiation by a truncated elliptical cylinder either totally submerged and resting on the seabed or partially immersed and floating on the free water surface was considered by Williams and Darwiche (1990). In their study, the expressions for the velocity potential were written in terms of infinite series involving Mathieu and modified Mathieu functions in elliptical coordinates. The analytical results revealed that, for the floating cylinder case, the values of the added-mass in pitch mode due to pitch motion did not vary significantly with changing eccentricity. Later, a similar analytical approach was utilized by Zhang and Williams (1996) to study the radiation problem by a horizontally submerged disk of elliptic cross section. Analytical study on the hydrodynamic of arrays of bottom mounted or truncated elliptical cylinders can be found in Chatjigeorgiou and Mavrakos (2010), and Chatjigeorgiou (2013), respectively.

All of the research works mentioned above are concerned with the vertical cylinder with either circular or elliptical cross section, and the corresponding analytical models are not able to deal with the more general radiation problem by a cylinder with a different cross section, e.g."rectangular" or "quasi-elliptical". Recently, Liu et al. (2016; 2017) solved the wave diffraction problem of a uniform (i.e. bottom mounted) and a truncated cylinder with non-circular/elliptical cross section, respectively. The Fourier series expansion method was used to describe the radius function of the cylinder surface and Bessel functions. The semi-analytical results of both wave excitation forces and water elevation around the cylinder agreed rather well with the numerical ones. Additionally, the problem of wave diffraction from a bottom-mounted cylinder was also considered by Disibüyük et al. (2017) with an asymptotic approach. The asymptotic solution of the wave run-up around the cylinder with cosine-type perturbation cross section provided good agreement with those by Liu et al. (2016).

In the present paper, we limit our attention to the solution of the radiation problem from a truncated cylinder with an arbitrary cross section. A semi-analytical model for solving such problem is presented based on linear potential flow theory. The Fourier series expansion method previously used by Liu et al. $(2016 ; 2017)$ for wave diffraction problem is applied to deal with the radius function associated terms. The rest of the paper is organized as follows. Section 2 presents the governing equations and boundary conditions of the radiation problem by a floating truncated cylinder of arbitrary cross section. Expressions and solutions of the radiated potentials are given in Section 3. Expressions of hydrodynamic coefficients are derived in Section 4. Upon validation with numerical modelling results, the semi-analytical model is applied to study the hydrodynamic characteristics of the cylinder with different cross sections, i.e. "circular", "cosine", "elliptical" and "quasi-elliptical", in Section 5. Conclusions are summarized in Section 6.

\section{Mathematical model}

Consider a floating vertical truncated cylinder with an arbitrary cross section oscillating time-harmonically in water of depth $h$ (Fig. 1). The draught of the cylinder is $d$. Let the Oxy plane be the horizontal mean water surface, and let $(r, \theta)$ be polar coordinates in the horizontal plane with the $O z$-axis pointing upwards. The rotation motion is assumed about the point $\left(0,0, z_{0}\right)$, which can also be used as a reference point to calculate the radiation moment acting on the cylinder. 
(a)
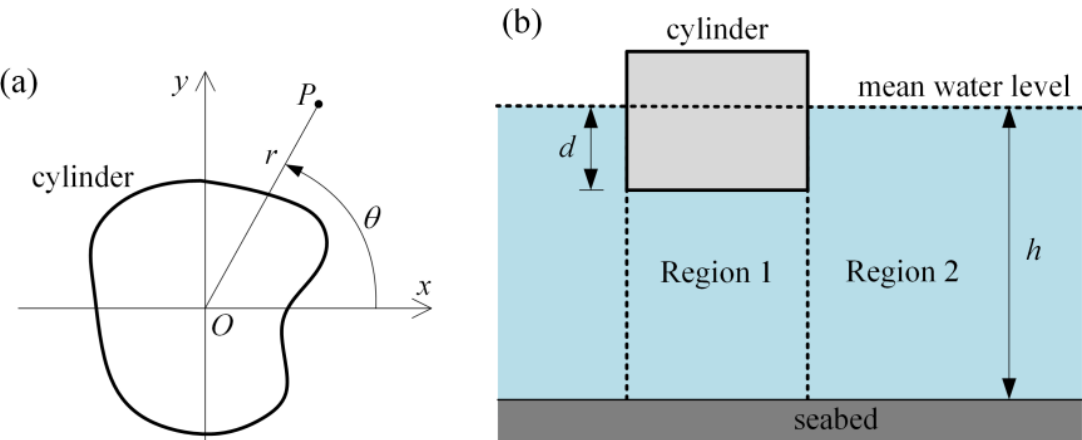

Fig. 1. Definition sketch: (a) plan view; (b) side view.

The shape of the cylinder cross section can be described in polar coordinate system $\operatorname{Or} \theta z$ as $r=R(\theta)$, which represents the radius of any point at cross section at $\theta$. To describe the unit normal vector at the side surface of the cylinder, the $S$ function is introduced as:

$$
S(r, \theta)=r-R(\theta),
$$

where $S=0$ represents the cross section as well, and the unit normal vector pointing into the water at the side surface in $\operatorname{Or} \theta z$ system and in $O x y z$ system can be written as Eq.(2a) and Eq.(2b), respectively:

$$
\vec{n}=\frac{1}{\sqrt{1+\left(\frac{1}{r} \frac{\partial S}{\partial \theta}\right)^{2}}}\left(\vec{r}+\frac{1}{r} \frac{\partial S}{\partial \theta} \vec{\theta}+0 \vec{z}\right)
$$

$$
\vec{n}=\frac{1}{\sqrt{1+\left(\frac{1}{r} \frac{\partial S}{\partial \theta}\right)^{2}}}\left(\left(\cos \theta-\frac{1}{r} \frac{\partial S}{\partial \theta} \sin \theta\right) \vec{i}+\left(\sin \theta+\frac{1}{r} \frac{\partial S}{\partial \theta} \cos \theta\right) \vec{j}+0 \vec{k}\right)
$$

With the same assumption employed in our previous study (Zheng and Zhang, 2015; 2016), fluid motion due to small oscillation of the cylinder can be described by the velocity potential $\phi(x, y, z, t)=\operatorname{Re}\left[\Phi(x, y, z) \mathrm{e}^{-\mathrm{i} \omega t}\right]$, where $t$ is the time, $\omega$ is the angular frequency of the cylinder's oscillation; $\Phi$ is a complex spatial velocity potential independent of time and satisfies the Laplace equation, $\mathrm{i}=\sqrt{-1} . \Phi$ can be decomposed in to six components as follows:

$$
\Phi=\sum_{i=1}^{6} \Phi_{\mathrm{R}}^{(i)} u_{i}
$$

where $\Phi_{\mathrm{R}}^{(i)}$ is the spatial velocity potential due to unit amplitude velocity oscillation of the truncated cylinder in $i$-th mode $(i=1,2, \ldots, 6$ denotes the motion mode of surge, sway, heave, roll, pitch and yaw, respectively), and $u_{i}$ represents the corresponding complex velocity amplitude of the oscillation motion in the same mode.

The governing equation and the boundary conditions for the radiated spatial potential $\Phi_{\mathrm{R}}^{(i)}$ can be written as follows:

1) The Laplace equation 


$$
\frac{\partial^{2} \Phi_{\mathrm{R}}^{(i)}}{\partial x^{2}}+\frac{\partial^{2} \Phi_{\mathrm{R}}^{(i)}}{\partial y^{2}}+\frac{\partial^{2} \Phi_{\mathrm{R}}^{(i)}}{\partial z^{2}}=0
$$

2) The linear free surface condition:

$$
\frac{\partial \Phi_{\mathrm{R}}^{(i)}}{\partial z}-\frac{\omega^{2}}{g} \Phi_{\mathrm{R}}^{(i)}=0, \quad z=0 \text { and } r \geq R(\theta)
$$

3) The non-penetrating condition on seabed bottom:

$$
\frac{\partial \Phi_{\mathrm{R}}^{(i)}}{\partial z}=0, \quad z=-h
$$

4) The body surface condition:

$$
\frac{\partial \Phi_{\mathrm{R}}^{(i)}}{\partial z}=\delta_{3, i}+\delta_{4, i} r \sin \theta-\delta_{5, i} r \cos \theta, \quad z=-d \quad \text { and } 0 \leq r \leq R(\theta)
$$

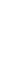

$$
r^{2} \frac{\partial \Phi_{\mathrm{R}}^{(i)}}{\partial r}+\frac{\partial S}{\partial \theta} \frac{\partial \Phi_{\mathrm{R}}^{(i)}}{\partial \theta}=F_{1}^{(i)}, \quad-d \leq z \leq 0 \text { and } r=R(\theta)
$$

9

where $\delta_{i, j}$ represents the Kronecker delta function, and

$$
F_{1}^{(i)}= \begin{cases}r^{2}\left(\cos \theta-\frac{\partial S}{r \partial \theta} \sin \theta\right), & i=1 \\ r^{2}\left(\sin \theta+\frac{\partial S}{r \partial \theta} \cos \theta\right), & i=2 \\ 0, & i=3 \\ -\left(z-z_{0}\right) r^{2}\left(\sin \theta+\frac{\partial S}{r \partial \theta} \cos \theta\right), & i=4 \\ \left(z-z_{0}\right) r^{2}\left(\cos \theta-\frac{\partial S}{r \partial \theta} \sin \theta\right), & i=5 \\ r^{2} \frac{\partial S}{\partial \theta}, & i=6\end{cases}
$$

5) The radiation condition at infinity:

$$
\sqrt{k_{0} r}\left(\frac{\partial \Phi_{\mathrm{R}}^{(i)}}{\partial r}-\mathrm{i} k_{0} \Phi_{\mathrm{R}}^{(i)}\right)=0, \quad r \rightarrow \infty,
$$

where $k_{0}$ is the wave number.

For the one vertical cylinder floating on the water surface, the whole fluid domain can be divided into two subdomains as indicated in Fig. 1: region 1, the interior domain beneath the cylinder, i.e. $r \leq R(\theta),-h \leq \mathrm{z} \leq-d$; region 2 , the exterior domain extending to infinity in the horizontal plane, i.e., $r \geq R(\theta)$ and $-h \leq \mathrm{z} \leq 0$. The radiated spatial potential in these subdomains are denoted by $\Phi_{\mathrm{R}, 1}^{(i)}$ and $\Phi_{\mathrm{R}, 2}^{(i)}$, respectively. 


\section{Solution to radiated potentials}

\subsection{Radiated spatial potentials in subdomains}

Following our previous research work (Zheng and Zhang, 2016), the radiated spatial potentials in different subdomains can be expressed as follows:

1) Region 1

$$
\Phi_{\mathrm{R}, 1}^{(i)}(r, \theta, z)=\Phi_{\mathrm{R}, \mathrm{p}}^{(i)}+\sum_{m=-\infty}^{\infty}\left[A_{m, 0}^{(i)}(r)^{|m|}+\sum_{l=1}^{\infty} A_{m, l}^{(i)} I_{m}\left(\beta_{l} r\right) \cos \left[\beta_{l}(z+h)\right]\right] \mathrm{e}^{\mathrm{i} m \theta},
$$

where $A_{m, l}^{(i)}$ is the unknown coefficient to be solved in Section 3.2; $I_{m}$ is the modified Bessel function of first kind and order $m ; \beta_{l}$ is the eigenvalue for interior region which is given by

$$
\beta_{l}=\frac{l \pi}{h-d}, \quad l=0,1,2,3, \ldots,
$$

$\Phi_{\mathrm{R}, \mathrm{p}}^{(i)}$ is a particular solution, the expression of which can be written in $\operatorname{Or} \theta z$ system as

$$
\Phi_{\mathrm{R}, \mathrm{p}}^{(i)}=\left\{\begin{array}{ll}
0, \quad i=1,2,6 \\
\frac{1}{4(h-d)}\left[2(z+h)^{2}-r^{2}\right], & i=3 \\
\frac{\sin \theta}{8(h-d)}\left[4 r(z+h)^{2}-r^{3}\right], & i=4 \\
\frac{\cos \theta}{8(h-d)}\left[r^{3}-4 r(z+h)^{2}\right], & i=5
\end{array},\right.
$$

2) Region 2

$$
\Phi_{\mathrm{R}, 2}^{(i)}(r, \theta, z)=\sum_{m=-\infty}^{\infty}\left[B_{m, 0}^{(i)} H_{m}\left(k_{0} r\right) \frac{Z_{0}(z)}{Z_{0}(0)}+\sum_{l=1}^{\infty} B_{m, l}^{(i)} K_{m}\left(k_{l} r\right) \frac{Z_{l}(z)}{Z_{l}(0)}\right] \mathrm{e}^{\mathrm{i} m \theta}
$$

where

$$
\begin{array}{cc}
Z_{0}(z)=N_{0}^{-1 / 2} \cosh \left[k_{0}(z+h)\right] ; & Z_{l}(z)=N_{l}^{-1 / 2} \cos \left[k_{l}(z+h)\right] ; \\
N_{0}=\frac{1}{2}\left[1+\frac{\sinh \left(2 k_{0} h\right)}{2 k_{0} h}\right] ; & N_{l}=\frac{1}{2}\left[1+\frac{\sin \left(2 k_{l} h\right)}{2 k_{l} h}\right] ;
\end{array}
$$

$B_{m, l}^{(i)}$ is the unknown coefficient to be solved in Section 3.2; $H_{m}$ is the Hankel function of first kind of order $m ; K_{m}$ is the modified Bessel function of second kind of order $m ; k_{l}$ is the eigenvalue for exterior region which is given by:

$$
\omega^{2}=-k_{l} g \tan \left(k_{l} h\right), \quad l=1,2,3, \ldots
$$

\subsection{Method of computation for coefficients}

The radiated spatial potentials as given in Eqs. (11) and (14) satisfy all the boundary conditions as shown in Eqs. (4-10), except those on the interface of the two subdomains at $r=R(\theta)$. 
1 The conditions of continuity of pressure and normal velocity at $r=R(\theta)$ can be used to determine

2 the unknown coefficients $A_{m, l}^{(i)}, B_{m, l}^{(i)}$ involved in Eqs. (11) and (14).

3 The continuity conditions of pressure and normal velocity are given as follows:

4 1) Continuity of pressure at the boundary $S=0$ :

6

7

$$
\left.\Phi_{\mathrm{R}, 2}^{(i)}\right|_{S=0}=\left.\Phi_{\mathrm{R}, 1}^{(i)}\right|_{S=0}, \quad-h<z<-d
$$

2) Continuity of normal velocity at the boundary $S=0$ :

$$
\left.\left(r^{2} \frac{\partial \Phi_{\mathrm{R}, 2}^{(i)}}{\partial r}+\frac{\partial S}{\partial \theta} \frac{\partial \Phi_{\mathrm{R}, 2}^{(i)}}{\partial \theta}\right)\right|_{S=0}=\left\{\begin{array}{ll}
\left.F_{1}^{(i)}\right|_{S=0}, & -d<z<0 \\
\left.\left(r^{2} \frac{\partial \Phi_{\mathrm{R}, 1}^{(i)}}{\partial r}+\frac{\partial S}{\partial \theta} \frac{\partial \Phi_{\mathrm{R}, 1}^{(i)}}{\partial \theta}\right)\right|_{S=0},-h<z<-d
\end{array} .\right.
$$

Inserting the expression of the radiated spatial potential into the boundary conditions above gives:$$
\left.\sum_{m=-\infty}^{\infty}\left[B_{m, 0}^{(i)} H_{m}\left(k_{0} r\right) \frac{Z_{0}(z)}{Z_{0}(0)}+\sum_{l=1}^{\infty} B_{m, l}^{(i)} K_{m}\left(k_{l} r\right) \frac{Z_{l}(z)}{Z_{l}(0)}\right] \mathrm{e}^{\mathrm{i} m \theta}\right|_{S=0}
$$$$
=\left.\Phi_{\mathrm{R}, \mathrm{p}}^{(i)}\right|_{S=0}+\left.\sum_{m=-\infty}^{\infty}\left[A_{m, 0}^{(i)}(r)^{|m|}+\sum_{l=1}^{\infty} A_{m, l}^{(i)} I_{m}\left(\beta_{l} r\right) \cos \left[\beta_{l}(z+h)\right]\right] \mathrm{e}^{\mathrm{i} m \theta}\right|_{S=0}, \quad-h<z<-d
$$

$$
\left.\sum_{m=-\infty}^{\infty}\left[B_{m, 0}^{(i)}\left(r^{2} k_{0} H_{m}^{\prime}\left(k_{0} r\right)+\mathrm{i} m H_{m}\left(k_{0} r\right) \frac{\partial S}{\partial \theta}\right) \frac{Z_{0}(z)}{Z_{0}(0)}+\sum_{l=1}^{\infty} B_{m, l}^{(i)}\left(r^{2} k_{l} K_{m}^{\prime}\left(k_{l} r\right)+\mathrm{i} m K_{m}\left(k_{l} r\right) \frac{\partial S}{\partial \theta}\right) \frac{Z_{l}(z)}{Z_{l}(0)}\right] \mathrm{e}^{\mathrm{i} m \theta}\right|_{S=0}
$$

$$
= \begin{cases}\left.F_{1}^{(i)}\right|_{S=0}, & -d<z<0, \\ \left.F_{2}^{(i)}\right|_{S=0}+\left.\sum_{m=-\infty}^{\infty}\left[A_{m, 0}^{(i)}\left(|m| r^{|m|+1}+\mathrm{i} m r^{|m|} \frac{\partial S}{\partial \theta}\right)+\sum_{l=1}^{\infty} A_{m, l}^{(i)}\left(r^{2} \beta_{l} I_{m}^{\prime}\left(\beta_{l} r\right)+\mathrm{i} m I_{m}\left(\beta_{l} r\right) \frac{\partial S}{\partial \theta}\right) \cos \beta_{l}(z+h)\right] \mathrm{e}^{\mathrm{i} m \theta}\right|_{S=0},-h<z<-d\end{cases}
$$

where $F_{2}^{(i)}=r^{2} \frac{\partial \Phi_{\mathrm{R}, \mathrm{p}}^{(i)}}{\partial r}+\frac{\partial S}{\partial \theta} \frac{\partial \Phi_{\mathrm{R}, \mathrm{p}}^{(i)}}{\partial \theta}$, and its expression in terms of the radius function of cross section can be written as:

$$
F_{2}^{(i)}=\frac{1}{8(h-d)}\left\{\begin{array}{lr}
0, & i=1,2,6 \\
-4 r^{3}, & i=3 \\
4(z+h)^{2} r\left(r \sin \theta+\frac{\partial S}{\partial \theta} \cos \theta\right)-r^{3}\left(3 r \sin \theta+\frac{\partial S}{\partial \theta} \cos \theta\right), & i=4 \\
-4(z+h)^{2} r\left(r \cos \theta-\frac{\partial S}{\partial \theta} \sin \theta\right)+r^{3}\left(3 r \cos \theta-\frac{\partial S}{\partial \theta} \sin \theta\right), & i=5
\end{array}\right.
$$

To solve the wave radiation problem, the functions relating to the radius function of the cylinder cross section i.e., $S=0$, are expanded into a Fourier series as follows:

$$
\left.H_{m}\left(k_{0} r\right)\right|_{S=0}=\sum_{q=-\infty}^{\infty} f_{m, 0, q}^{H} \mathrm{e}^{\mathrm{i} q \theta} ;\left.\left(r^{2} k_{0} H_{m}^{\prime}\left(k_{0} r\right)+\mathrm{i} m H_{m}\left(k_{0} r\right) \frac{\partial S}{\partial \theta}\right)\right|_{S=0}=\sum_{q=-\infty}^{\infty} f_{m, 0, q}^{H^{\prime}} \mathrm{e}^{\mathrm{i} q \theta},
$$

$$
\left.K_{m}\left(k_{l} r\right)\right|_{S=0}=\sum_{q=-\infty}^{\infty} f_{m, l, q}^{K} \mathrm{e}^{\mathrm{i} q \theta} ;\left.\left(r^{2} k_{l} K_{m}^{\prime}\left(k_{l} r\right)+\mathrm{i} m K_{m}\left(k_{l} r\right) \frac{\partial S}{\partial \theta}\right)\right|_{S=0}=\sum_{q=-\infty}^{\infty} f_{m, l, q}^{K^{\prime}} \mathrm{e}^{\mathrm{i} q \theta}
$$

$$
\left.I_{m}\left(\beta_{l} r\right)\right|_{S=0}=\sum_{q=-\infty}^{\infty} \tilde{f}_{m, l, q}^{I} \mathrm{e}^{\mathrm{i} q \theta} ;\left.\left(r^{2} \beta_{l} I_{m}^{\prime}\left(\beta_{l} r\right)+\mathrm{i} m I_{m}\left(\beta_{l} r\right) \frac{\partial S}{\partial \theta}\right)\right|_{S=0}=\sum_{q=-\infty}^{\infty} \tilde{f}_{m, l, q}^{I^{\prime}} \mathrm{e}^{\mathrm{i} q \theta}
$$


14

$$
\left.r^{|m|}\right|_{S=0}=\sum_{q=-\infty}^{\infty} f_{m, 0, q}^{R} \mathrm{e}^{\mathrm{i} q \theta} ;\left.\left(|m| r^{|m|+1}+\mathrm{i} m r^{|m|} \frac{\partial S}{\partial \theta}\right)\right|_{S=0}=\sum_{q=-\infty}^{\infty} \tilde{f}_{m, 0, q}^{R} \mathrm{e}^{\mathrm{i} q \theta}
$$

$$
\left.\left(r \cos \theta-\frac{\partial S}{\partial \theta} \sin \theta\right)\right|_{S=0}=\sum_{q=-\infty}^{\infty} f_{q}^{(1)} \mathrm{e}^{\mathrm{i} q \theta} ;\left.\left(r \sin \theta+\frac{\partial S}{\partial \theta} \cos \theta\right)\right|_{S=0}=\sum_{q=-\infty}^{\infty} f_{q}^{(2)} \mathrm{e}^{\mathrm{i} q \theta}
$$

$$
\left.r\left(r \sin \theta+\frac{\partial S}{\partial \theta} \cos \theta\right)\right|_{S=0}=\sum_{q=-\infty}^{\infty} f_{q}^{(4)} \mathrm{e}^{\mathrm{i} q \theta} ;\left.r^{3}\left(3 r \sin \theta+\frac{\partial S}{\partial \theta} \cos \theta\right)\right|_{S=0}=\sum_{q=-\infty}^{\infty} f_{q}^{4^{\prime}} \mathrm{e}^{\mathrm{i} q \theta}
$$

$$
\left.r\left(r \cos \theta-\frac{\partial S}{\partial \theta} \sin \theta\right)\right|_{S=0}=\sum_{q=-\infty}^{\infty} f_{q}^{(5)} \mathrm{e}^{\mathrm{i} q \theta} ;\left.r^{3}\left(3 r \cos \theta-\frac{\partial S}{\partial \theta} \sin \theta\right)\right|_{S=0}=\sum_{q=-\infty}^{\infty} f_{q}^{5^{\prime}} \mathrm{e}^{\mathrm{i} q \theta}
$$

$$
\left.r \frac{\partial S}{\partial \theta}\right|_{S=0}=\sum_{q=-\infty}^{\infty} f_{q}^{(6)} \mathrm{e}^{\mathrm{i} q \theta} ;\left.r^{2} \frac{\partial S}{\partial \theta}\right|_{S=0}=\sum_{q=-\infty}^{\infty} f_{q}^{6^{\prime}} \mathrm{e}^{\mathrm{i} q \theta}
$$

All these Fourier coefficients, such as $f_{m, 0, q}^{H}, f_{m, 0, q}^{H^{\prime}}, f_{m, l, q}^{K}, f_{m, l, q}^{K^{\prime}}$ and so on as shown in Eqs.(23-30), can be easily obtained by using:

$$
\chi_{q}=\frac{1}{2 \pi} \int_{0}^{2 \pi} \psi(\theta) \mathrm{e}^{-\mathrm{i} q \theta} \mathrm{d} \theta
$$

in which $\chi_{q}$ and $\psi(\theta)$ represent the Fourier coefficients and functions to be expanded, respectively.

With the utilization of these Fourier expansions as given in Eqs. (23-30), Eqs. (20-21) can be rewritten as:

$$
\begin{aligned}
& \sum_{m=-\infty}^{\infty}\left[B_{m, 0}^{(i)} \frac{Z_{0}(z)}{Z_{0}(0)} \sum_{q=-\infty}^{\infty} f_{m, 0, q}^{H} \mathrm{e}^{\mathrm{i} q \theta}+\sum_{l=1}^{\infty} B_{m, l}^{(i)} \frac{Z_{l}(z)}{Z_{l}(0)} \sum_{q=-\infty}^{\infty} f_{m, l, q}^{K} \mathrm{e}^{\mathrm{i} q \theta}\right] \mathrm{e}^{\mathrm{i} m \theta} \\
& =\left.\Phi_{\mathrm{R}, \mathrm{p}}^{(i)}\right|_{S=0}+\sum_{m=-\infty}^{\infty}\left[A_{m, 0}^{(i)} \sum_{q=-\infty}^{\infty} f_{m, 0, q}^{R} \mathrm{e}^{\mathrm{i} q \theta}+\sum_{l=1}^{\infty} A_{m, l}^{(i)} \cos \left[\beta_{l}(z+h)\right] \sum_{q=-\infty}^{\infty} \tilde{f}_{m, l, q}^{I} \mathrm{e}^{\mathrm{i} q \theta}\right] \mathrm{e}^{\mathrm{i} m \theta}, \\
& \sum_{m=-\infty}^{\infty}\left[B_{m, 0}^{(i)} \frac{Z_{0}(z)}{Z_{0}(0)} \sum_{q=-\infty}^{\infty} f_{m, 0, q}^{H^{\prime}} \mathrm{e}^{\mathrm{i} q \theta}+\sum_{l=1}^{\infty} B_{m, l}^{(i)} \frac{Z_{l}(z)}{Z_{l}(0)} \sum_{q=-\infty}^{\infty} f_{m, l, q}^{K^{\prime}} \mathrm{e}^{\mathrm{i} q \theta}\right] \mathrm{e}^{\mathrm{i} m \theta}
\end{aligned}
$$

$$
=\left\{\begin{array}{l}
\left.F_{1}^{(i)}\right|_{S=0} \\
\left.F_{2}^{(i)}\right|_{S=0}+\sum_{m=-\infty}^{\infty}\left[A_{m, 0}^{(i)} \sum_{q=-\infty}^{\infty} \tilde{f}_{m, 0, q}^{R} \mathrm{e}^{\mathrm{i} q \theta}+\sum_{l=1}^{\infty} A_{m, l}^{(i)} \sum_{q=-\infty}^{\infty} \tilde{f}_{m, l, q}^{I^{\prime}} \mathrm{e}^{\mathrm{i} q \theta} \cos \left[\beta_{l}(z+h)\right] \mathrm{e}^{\mathrm{i} m \theta}\right.
\end{array}\right.
$$

where $\left.\Phi_{\mathrm{R}, \mathrm{p}}^{(i)}\right|_{S=0},\left.\quad F_{1}^{(i)}\right|_{S=0}$ and $\left.F_{2}^{(i)}\right|_{S=0}$ can be expressed in terms of the Fourier coefficients as: 


$$
\left.\Phi_{\mathrm{R}, \mathrm{p}}^{(i)}\right|_{S=0}=\left\{\begin{array}{lr}
0, & i=1,2,6 \\
\frac{1}{4(h-d)}\left[2(z+h)^{2}-\sum_{q=-\infty}^{\infty} f_{2,0, q}^{R} \mathrm{e}^{\mathrm{i} q \theta}\right], & i=3 \\
\frac{1}{16 \mathrm{i}(h-d)}\left[4(z+h)^{2} \sum_{q=-\infty}^{\infty}\left(f_{1,0, q-1}^{R}-f_{1,0, q+1}^{R}\right) \mathrm{e}^{\mathrm{i} q \theta}-\sum_{q=-\infty}^{\infty}\left(f_{3,0, q-1}^{R}-f_{3,0, q+1}^{R}\right) \mathrm{e}^{\mathrm{i} q \theta}\right], & i=4, \\
\frac{-1}{16(h-d)}\left[4(z+h)^{2} \sum_{q=-\infty}^{\infty}\left(f_{1,0, q-1}^{R}+f_{1,0, q+1}^{R}\right) \mathrm{e}^{\mathrm{i} q \theta}-\sum_{q=-\infty}^{\infty}\left(f_{3,0, q-1}^{R}+f_{3,0, q+1}^{R}\right) \mathrm{e}^{\mathrm{i} q \theta}\right], & i=5
\end{array}\right.
$$

$$
\begin{aligned}
& \left.F_{1}^{(i)}\right|_{S=0}= \begin{cases}\sum_{q=-\infty}^{\infty} f_{q}^{(5)} \mathrm{e}^{\mathrm{i} q \theta}, & i=1 \\
\sum_{q=-\infty}^{\infty} f_{q}^{(4)} \mathrm{e}^{\mathrm{i} q \theta}, & i=2 \\
0, & i=3 \\
-\left(z-z_{0}\right) \sum_{q=-\infty}^{\infty} f_{q}^{(4)} \mathrm{e}^{\mathrm{i} q \theta}, & i=4 \\
\left(z-z_{0}\right) \sum_{q=-\infty}^{\infty} f_{q}^{(5)} \mathrm{e}^{\mathrm{i} q \theta}, & i=5 \\
\sum_{q=-\infty}^{\infty} f_{q}^{6^{\prime}} \mathrm{e}^{\mathrm{i} q \theta}, & i=6\end{cases} \\
& \left.F_{2}^{(i)}\right|_{S=0}=\frac{1}{8(h-d)}\left\{\begin{array}{lr}
0, & i=1,2,6 \\
-4 \sum_{q=-\infty}^{\infty} f_{3,0, q}^{R} \mathrm{e}^{\mathrm{i} q \theta}, & i=3 \\
4(z+h)^{2} \sum_{q=-\infty}^{\infty} f_{q}^{(4)} \mathrm{e}^{\mathrm{i} q \theta}-\sum_{q=-\infty}^{\infty} f_{q}^{4^{\prime}} \mathrm{e}^{\mathrm{i} q \theta}, & i=4 \\
-4(z+h)^{2} \sum_{q=-\infty}^{\infty} f_{q}^{(5)} \mathrm{e}^{\mathrm{i} q \theta}+\sum_{q=-\infty}^{\infty} f_{q}^{5^{\prime}} \mathrm{e}^{\mathrm{i} q \theta}, & i=5
\end{array} .\right.
\end{aligned}
$$

Using the orthogonality characteristics of $Z_{l}(z), \cos \left[\beta_{l}(z+h)\right]$ and $\mathrm{e}^{\mathrm{i} m \theta}$, the unknown coefficients $A_{m, l}^{(i)}$ and $B_{m, l}^{(i)}$ can be calculated by solving a complex linear matrix equation. Deduction process of the formulas and calculation of $A_{m, l}^{(i)}$ and $B_{m, l}^{(i)} \quad$ are given in Appendix A.

\section{Hydrodynamic coefficients}

The complex amplitudes of the radiation force exerted on the truncated cylinder in $j$-th mode due to a unit amplitude velocity oscillation of itself in $i$-th mode can be written in terms of hydrodynamic coefficients, i.e. added-mass $a_{j, i}$ and radiation damping $c_{j, i}$, as:

$$
F_{\mathrm{R}, i}^{(j)}=-\mathrm{i} \omega \rho \int_{S_{\mathrm{w}}} \Phi_{\mathrm{R}}^{(i)} n_{j} \mathrm{~d} s=\mathrm{i} \omega a_{j, i}-c_{j, i},
$$

where $S_{\mathrm{w}}$ represents the wet surface of the truncated cylinder, $n_{j}$ is the generalized normal with $n_{1}=n_{x}, n_{2}=n_{y}, n_{3}=n_{z}, n_{4}=-\left(z-z_{0}\right) n_{y}+y n_{z}, n_{5}=\left(z-z_{0}\right) n_{x}-x n_{z}, n_{6}=-y n_{x}+x n_{y}, \quad \vec{n}=n_{x} \vec{i}+n_{y} \vec{j}+n_{z} \vec{k}$ is the unit normal vector directed into the fluid domain at the considered cylinder surface as given in Eq. (2b). 
1

8

10

12

$$
\begin{gathered}
\left.\mathrm{i} \omega \rho \int_{0}^{2 \pi} \int_{0}^{R} \Phi_{\mathrm{R}, \mathrm{p}}^{(i)}\right|_{z=-d} r \mathrm{~d} r \mathrm{~d} \theta \\
=\pi \mathrm{i} \omega \rho\left\{\begin{array}{l}
0, \quad i=1,2,6 \\
\frac{1}{2(h-d)}\left[(h-d)^{2} f_{2,0,0}^{R}-\frac{1}{4} f_{4,0,0}^{R}\right], \quad i=3 \\
\frac{1}{8 \mathrm{i}(h-d)}\left[\frac{4}{3}(h-d)^{2}\left(f_{3,0,-1}^{R}-f_{3,0,1}^{R}\right)-\frac{1}{5}\left(f_{5,0,-1}^{R}-f_{5,0,1}^{R}\right)\right], \quad i=4 \\
\frac{1}{8(h-d)}\left[\frac{1}{5}\left(f_{5,0,-1}^{R}+f_{5,0,1}^{R}\right)-\frac{4}{3}(h-d)^{2}\left(f_{3,0,-1}^{R}+f_{3,0,1}^{R}\right)\right], \quad i=5
\end{array}\right.
\end{gathered}
$$

where

2) Radiation force in $y$ direction (sway mode)

$$
\begin{aligned}
& F_{\mathrm{R}, i}^{(2)}=-\left.\mathrm{i} \omega \rho \int_{0}^{2 \pi} \int_{-d}^{0}\left[\left(r \sin \theta+\frac{\partial S}{\partial \theta} \cos \theta\right) \Phi_{\mathrm{R}, 2}^{(i)}\right]\right|_{S=0} \mathrm{~d} z \mathrm{~d} \theta \\
& =-2 \pi \mathrm{i} \omega \rho \sum_{m=-\infty}^{\infty}\left[B_{m, 0}^{(i)} \sum_{q=-\infty}^{\infty} f_{-m-q}^{(2)} f_{m, 0, q}^{H} Z t_{0}+\sum_{l=1}^{\infty} B_{m, l}^{(i)} \sum_{q=-\infty}^{\infty} f_{-m-q}^{(2)} f_{m, l, q}^{K} Z t_{l}\right]
\end{aligned}
$$

3) Radiation force in $z$ direction (heave mode)

$$
F_{\mathrm{R}, i}^{(3)}=\left.\mathrm{i} \omega \rho \int_{0}^{2 \pi} \int_{0}^{R} \Phi_{\mathrm{R}, 1}^{(i)}\right|_{z=-d} r \mathrm{~d} r \mathrm{~d} \theta=\left.\mathrm{i} \omega \rho \int_{0}^{2 \pi} \int_{0}^{R} \Phi_{\mathrm{R}, \mathrm{p}}^{(i)}\right|_{z=-d} r \mathrm{~d} r \mathrm{~d} \theta
$$

$$
+2 \mathrm{i} \pi \omega \rho \sum_{m=-\infty}^{\infty}\left(A_{m, 0}^{(i)} \frac{f_{|m|+2,0,-m}^{R}}{|m|+2}\right)+\mathrm{i} \omega \rho \int_{0}^{2 \pi} \int_{0}^{R} \sum_{m=-\infty}^{\infty} \sum_{l=1}^{\infty} A_{m, l}^{(i)}(-1)^{l} \mathrm{e}^{\mathrm{i} m \theta} r I_{m}\left(\beta_{l} r\right) \mathrm{d} r \mathrm{~d} \theta
$$

where 
1

$$
\begin{aligned}
& F_{\mathrm{R}, i}^{(4)}=\left.\mathrm{i} \omega \rho \int_{0}^{2 \pi} \int_{-d}^{0}\left[\left(r \sin \theta+\frac{\partial S}{\partial \theta} \cos \theta\right) \Phi_{\mathrm{R}, 2}^{(i)}\right]\right|_{S=0}\left(z-z_{0}\right) \mathrm{d} z \mathrm{~d} \theta+\left.\mathrm{i} \omega \rho \int_{0}^{2 \pi} \int_{0}^{R} \Phi_{\mathrm{R}, 1}^{(i)}\right|_{z=-d} r \sin \theta r \mathrm{~d} r \mathrm{~d} \theta \\
& =2 \pi \mathrm{i} \omega \rho \sum_{m=-\infty}^{\infty}\left[B_{m, 0}^{(i)} \sum_{q=-\infty}^{\infty} f_{-m-q}^{(2)} f_{m, 0, q}^{H} Z Z t_{0}+\sum_{l=1}^{\infty} B_{m, l}^{(i)} \sum_{q=-\infty}^{\infty} f_{-m-q}^{(2)} f_{m, l, q}^{K} Z Z t_{l}\right] \\
& +\pi \omega \rho \sum_{m=-\infty}^{\infty}\left[A_{m, 0}^{(i)} \frac{f_{|m|+3,0,-m-1}^{R}-f_{|m|+3,0,-m+1}^{R}}{|m|+3}\right]+\mathrm{i} \omega \rho \int_{0}^{2 \pi} \int_{0}^{R} \sum_{m=-\infty}^{\infty} \sum_{l=1}^{\infty} A_{m, l}^{(i)} I_{m}\left(\beta_{l} r\right)(-1)^{l} \mathrm{e}^{\mathrm{i} m \theta} r \sin \theta r \mathrm{~d} r \mathrm{~d} \theta \\
& +\left.\mathrm{i} \omega \rho \int_{0}^{2 \pi} \int_{0}^{R} \Phi_{\mathrm{R}, \mathrm{p}}^{(i)}\right|_{z=-d} r \sin \theta r \mathrm{~d} r \mathrm{~d} \theta
\end{aligned}
$$

2 where

3

$$
\begin{gathered}
Z Z t_{l}=\int_{-d}^{0} \frac{Z_{l}(z)}{Z_{l}(0)}\left(z-z_{0}\right) \mathrm{d} z \\
=\left\{\begin{array}{l}
\frac{k_{0}\left(d+z_{0}\right) \sinh \left[k_{0}(h-d)\right]-\cosh \left(k_{0} h\right)+\cosh \left[k_{0}(h-d)\right]-k_{0} z_{0} \sinh \left(k_{0} h\right)}{k_{0}^{2} \cosh \left(k_{0} h\right)}, \quad l=0, \\
\frac{k_{l}\left(d+z_{0}\right) \sin \left[k_{l}(h-d)\right]+\cos \left(k_{l} h\right)-\cos \left[k_{l}(h-d)\right]-k_{l} z_{0} \sin \left(k_{l} h\right)}{k_{l}^{2} \cos \left(k_{l} h\right)}, \quad l=1,2,3, \cdots
\end{array}\right.
\end{gathered}
$$

$4 \quad$ and the part contributed by the special radiated spatial potential can be expressed as $\left.\mathrm{i} \omega \rho \int_{0}^{2 \pi} \int_{0}^{R} \Phi_{\mathrm{R}, \mathrm{p}}^{(i)}\right|_{z=-d} r \sin \theta r \mathrm{~d} r \mathrm{~d} \theta$

5

$$
=\pi \mathrm{i} \omega \rho\left\{\begin{array}{lc}
0, & i=1,2,6 \\
\frac{1}{4 \mathrm{i}(h-d)}\left[\frac{2}{3}(h-d)^{2}\left(f_{3,0,-1}^{R}-f_{3,0,1}^{R}\right)-\frac{1}{5}\left(f_{5,0,-1}^{R}-f_{5,0,1}^{R}\right)\right], & i=3 \\
\frac{1}{16(h-d)}\left[\frac{1}{6}\left(f_{6,0,-2}^{R}+f_{6,0,2}^{R}-2 f_{6,0,0}^{R, n}\right)-(h-d)^{2}\left(f_{4,0,-2}^{R}+f_{4,0,2}^{R}-2 f_{4,0,0}^{R}\right)\right], & i=4 \\
\frac{1}{16 \mathrm{i}(h-d)}\left[\frac{1}{6}\left(f_{6,0,-2}^{R}-f_{6,0,2}^{R}\right)-(h-d)^{2}\left(f_{4,0,-2}^{R}-f_{4,0,2}^{R}\right)\right], & i=5
\end{array} .\right.
$$

6 5) Radiation force moment about $y$ axis at the rotation center of the cylinder (pitch mode)

7

$$
\begin{aligned}
& F_{\mathrm{R}, i}^{(5)}=-\left.\mathrm{i} \omega \rho \int_{0}^{2 \pi} \int_{-d}^{0}\left[\left(r \cos \theta-\frac{\partial S}{\partial \theta} \sin \theta\right) \Phi_{\mathrm{R}, 2}^{(i)}\right]\right|_{S=0}\left(z-z_{0}\right) \mathrm{d} z \mathrm{~d} \theta-\left.\mathrm{i} \omega \rho \int_{0}^{2 \pi} \int_{0}^{R} \Phi_{\mathrm{R}, 1}^{(i)}\right|_{z=-d} r \cos \theta r \mathrm{~d} r \mathrm{~d} \theta \\
& =-2 \pi \mathrm{i} \omega \rho \sum_{m=-\infty}^{\infty}\left[B_{m, 0}^{(i)} \sum_{q=-\infty}^{\infty} f_{-m-q}^{(1)} f_{m, 0, q}^{H} Z Z t_{0}+\sum_{l=1}^{\infty} B_{m, l}^{(i)} \sum_{q=-\infty}^{\infty} f_{-m-q}^{(1)} f_{m, l, q}^{K} Z Z t_{l}\right] \\
& -\pi \mathrm{i} \omega \rho \sum_{m=-\infty}^{\infty}\left[A_{m, 0}^{(i)} \frac{\left.f_{|m|+3,0,-m-1}^{R}+f_{|m|+3,0,-m+1}^{R}\right]-\mathrm{i} \omega \rho \int_{0}^{2 \pi} \int_{0}^{R} \sum_{m=-\infty}^{\infty} \sum_{l=1}^{\infty} A_{m, l}^{(i)} I_{m}\left(\beta_{l} r\right)(-1)^{l} \mathrm{e}^{\mathrm{i} m \theta} r \cos \theta r \mathrm{~d} r \mathrm{~d} \theta}{|m|+3},\right. \\
& -\left.\mathrm{i} \omega \rho \int_{0}^{2 \pi} \int_{0}^{R} \Phi_{\mathrm{R}, \mathrm{p}}^{(i)}\right|_{z=-d} r \cos \theta r \mathrm{~d} r \mathrm{~d} \theta
\end{aligned}
$$

8 and the part contributed by the special radiated spatial potential can be expressed as 


$$
-\left.\mathrm{i} \omega \rho \int_{0}^{2 \pi} \int_{0}^{R} \Phi_{\mathrm{R}, \mathrm{p}}^{(i)}\right|_{z=-d} r \cos \theta r \mathrm{~d} r \mathrm{~d} \theta
$$

$$
=-\pi \mathrm{i} \omega \rho\left\{\begin{array}{lr}
0, & i=1,2,6 \\
\frac{1}{4(h-d)}\left[\frac{2}{3}(h-d)^{2}\left(f_{3,0,-1}^{R}+f_{3,0,1}^{R}\right)-\frac{1}{5}\left(f_{5,0,-1}^{R}+f_{5,0,1}^{R}\right)\right], & i=3 \\
\frac{1}{16 \mathrm{i}(h-d)}\left[(h-d)^{2}\left(f_{4,0,-2}^{R}-f_{4,0,2}^{R}\right)-\frac{1}{6}\left(f_{6,0,-2}^{R}-f_{6,0,2}^{R}\right)\right], & i=4 \\
\frac{1}{16(h-d)}\left[\frac{1}{6}\left(f_{6,0,-2}^{R}+f_{6,0,2}^{R}+2 f_{6,0,0}^{R}\right)-(h-d)^{2}\left(f_{4,0,-2}^{R}+f_{4,0,2}^{R}+2 f_{4,0,0}^{R}\right)\right], & i=5
\end{array} .\right.
$$

1

6) Radiation force moment about $z$ axis at the rotation center of the cylinder (yaw mode)

3

$$
\begin{aligned}
F_{\mathrm{R}, i}^{(6)} & =-\left.\mathrm{i} \omega \rho \int_{0}^{2 \pi} \int_{-d}^{0}\left[r \frac{\partial S}{\partial \theta} \Phi_{\mathrm{R}, 2}^{(i)}\right]\right|_{S=0} \mathrm{~d} z \mathrm{~d} \theta \\
& =-2 \pi \mathrm{i} \omega \rho \sum_{m=-\infty}^{\infty}\left[B_{m, 0}^{(i)} \sum_{q=-\infty}^{\infty} f_{-m-q}^{(6)} f_{m, 0, q}^{H} Z t_{0}+\sum_{l=1}^{\infty} B_{m, l}^{(i)} \sum_{q=-\infty}^{\infty} f_{-m-q}^{(6)} f_{m, l, q}^{K} Z t_{l}\right]
\end{aligned}
$$

\section{Results and discussion}

\subsection{Model validation}

The vertical truncated cylinder with cosine-type radius function used by Liu et al. (2017) is adopted here as a case study of the wave radiation problem. A numerical simulation of the same case is also conducted using commercial software (ANSYS AQWA) based on the Boundary Element Method as a comparison to validate the present semi-analytical model. Figure 2 shows the vertical truncated cylinder with cosine-type radius function and the surface mesh used in numerical model. The rotation center of the cylinder is chosen as $\left(r=0, z_{0}=0\right)$ for this truncated cylinder with cosine-type radius function and any other cases to be studied below as well.

The dimensionless quantities of the added-mass and radiation damping coefficients are defined by:

$$
\bar{a}_{j, i}=\frac{a_{j, i}}{\rho S_{0}^{p} d} ; \bar{c}_{j, i}=\frac{c_{j, i}}{\omega \rho S_{0}^{p} d},
$$

where $S_{0}$ is the area of the cylinder cross section, $p=1$ for $i, j=1,2,3$; $p=2$ for $i, j=4,5,6 ; p=1.5$ for $i=1,2,3$ and $j=4,5,6$, and for $i=4,5,6$ and $j=1,2,3$ as well. 


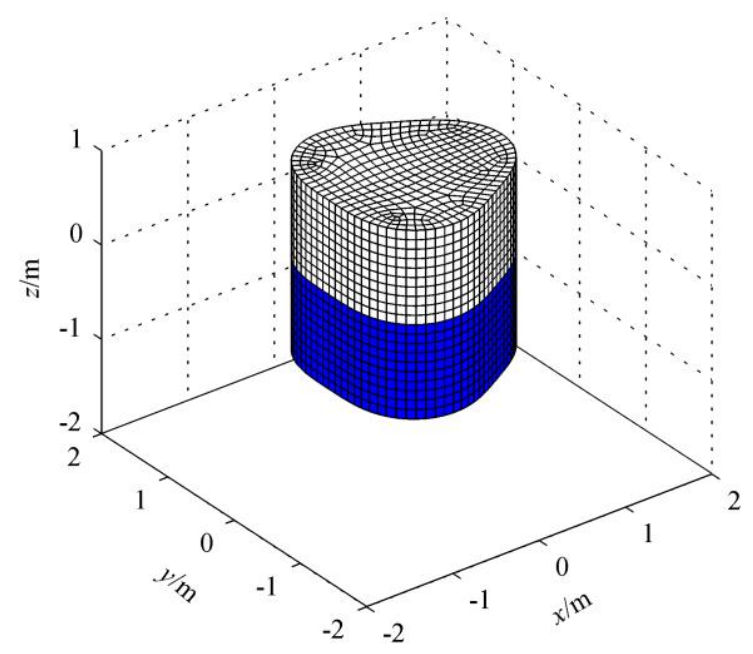

2 3

4

$7 \quad F_{\mathrm{R}, 1}^{(2)}=F_{\mathrm{R}, 1}^{(4)}=F_{\mathrm{R}, 1}^{(6)}=F_{\mathrm{R}, 3}^{(2)}=F_{\mathrm{R}, 3}^{(4)}=F_{\mathrm{R}, 3}^{(6)}=F_{\mathrm{R}, 5}^{(2)}=F_{\mathrm{R}, 5}^{(4)}=F_{\mathrm{R}, 5}^{(6)}=0$. Moreover, due to the symmetriy

8 property of the radius function in this case relative to the vertical centre line, i.e., $r=0$, we have $9 \quad F_{\mathrm{R}, 3}^{(1)}=F_{\mathrm{R}, 3}^{(2)}=F_{\mathrm{R}, 3}^{(4)}=F_{\mathrm{R}, 3}^{(5)}=0$. The reciprocity relation $F_{\mathrm{R}, i}^{(j)}=F_{\mathrm{R}, j}^{(i)}$ is satisfied for the wave 10 radiation problem from any marine structure (Falnes,2002). Therefore, the only nonvanishing 11 off-diagonal elements of the radiation hydrodynamic matrix are $F_{\mathrm{R}, 5}^{(1)}=F_{\mathrm{R}, 1}^{(5)}$ and $F_{\mathrm{R}, 4}^{(2)}=F_{\mathrm{R}, 2}^{(4)}$.

12 The normalised added-mass and radiation damping corresponding to the nonvanishing $F_{\mathrm{R}, i}^{(j)}$ in the 13 frequency domain as a function of $k h$ are plotted in Figs. 3 and 4, respectively.
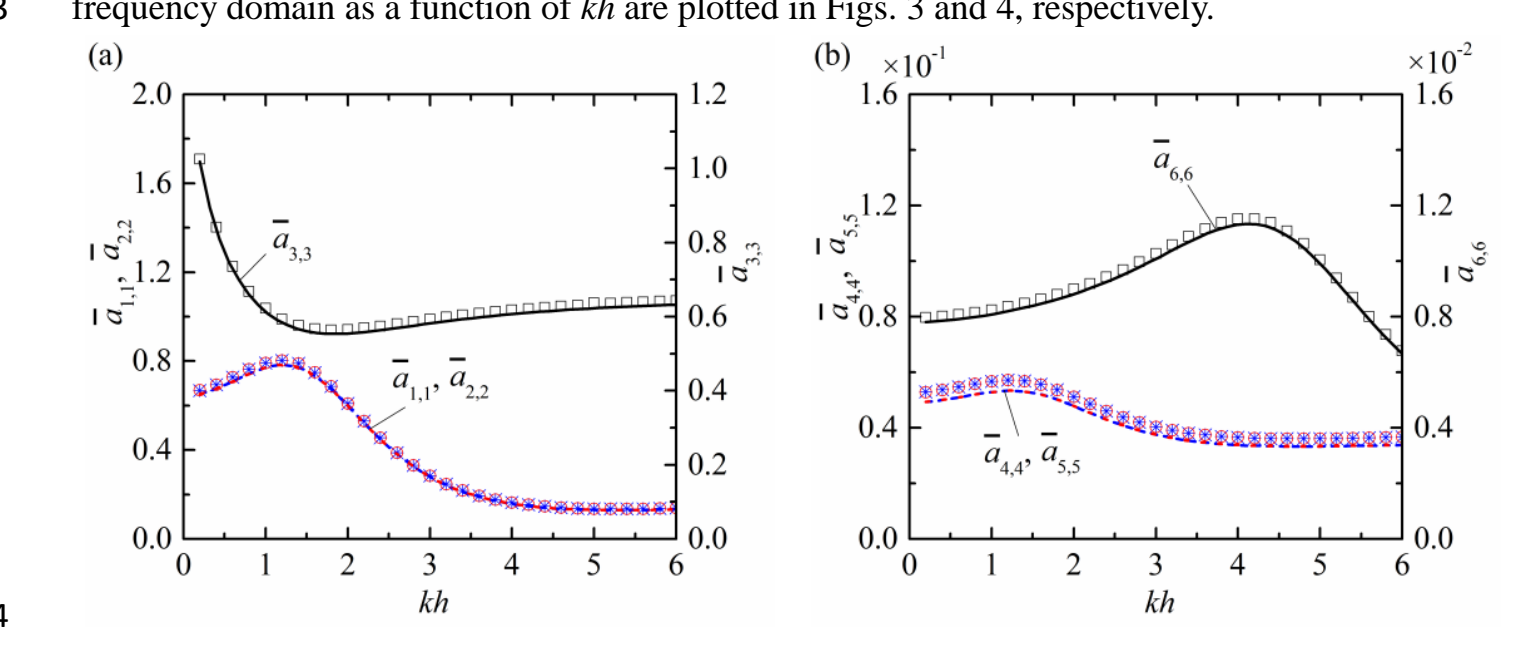

\section{(a)}

Fig. 2. Top view of the truncated cylinder with $h=2.0 \mathrm{~m}, R(\theta)=0.5 h[1+0.1 \cos (3 \theta)], d=0.5 h$ and the
6 of $y$, whereas $\Phi_{\mathrm{R}}^{(1)}, \Phi_{\mathrm{R}}^{(3)}$ and $\Phi_{\mathrm{R}}^{(5)}$ are even functions. Hence surface mesh used in numerical model.
Note in this case, $y=0$ is a plane of symmetry, then $n_{2}, n_{4}$ and $n_{6}$ in Eq. (37) are odd functions th 


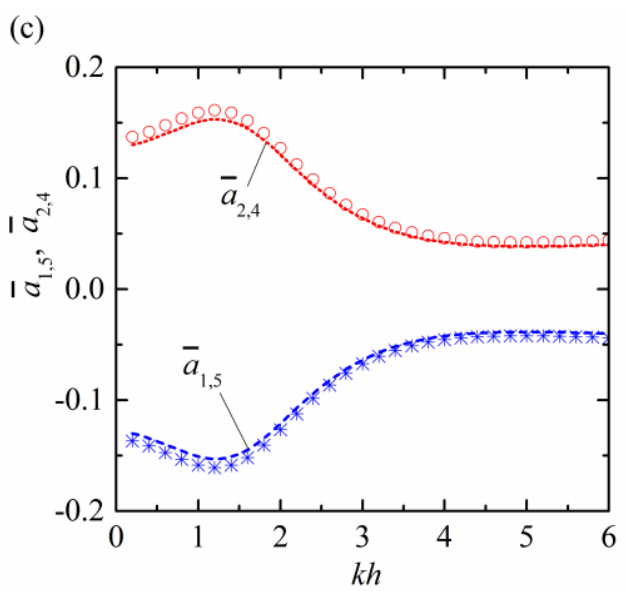

7

(a)

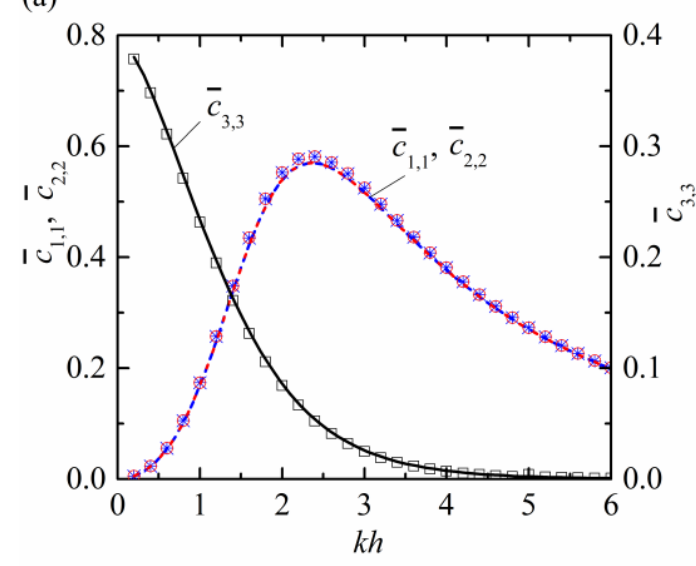

(c)
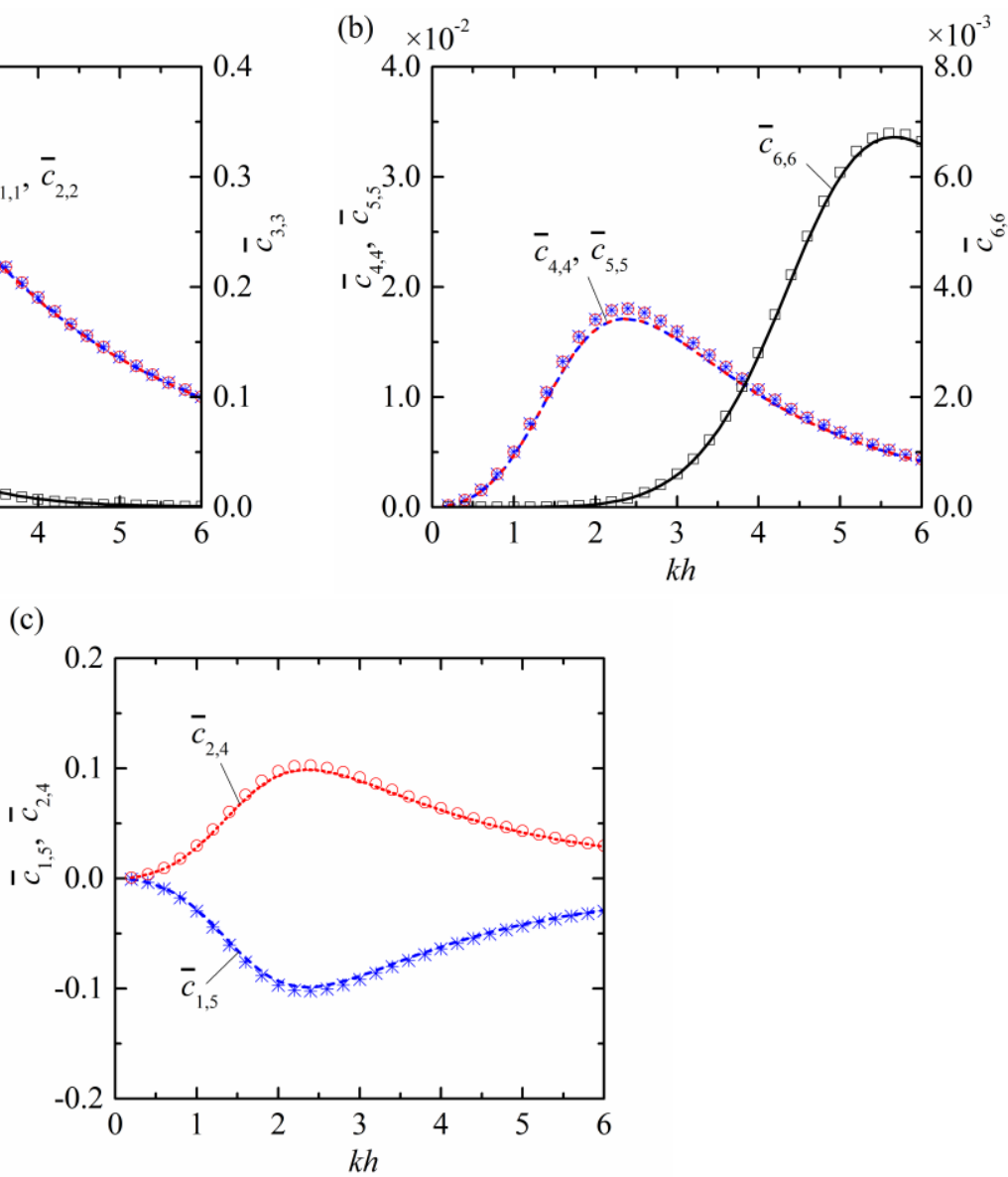

Fig. 3. Comparison of present semi-analytical results with numerical results in terms of normalised added-mass as a function of $k h$ for $R(\theta)=0.5 h[1+0.1 \cos (3 \theta)], d=0.5 h$ : (a) $\bar{a}_{1,1}, \bar{a}_{2,2}$ and $\bar{a}_{3,3} ;$ (b) $\bar{a}_{4,4}, \bar{a}_{5,5}$ and $\bar{a}_{6,6} ;$ (c) $\bar{a}_{1,5}$ and $\bar{a}_{2,4}$. Lines $(-\ldots \ldots, \ldots \ldots \ldots \ldots$, and $-\ldots$ ) represent the present semi-analytical results, and symbols $(*, \circ$, and $\square)$ denote the numerical results. 
$\bar{c}_{2,2}$ and $\bar{c}_{3,3} ;$ (b) $\bar{c}_{4,4}, \bar{c}_{5,5}$ and $\bar{c}_{6,6} ;$ (c) $\bar{c}_{1,5}$ and $\bar{c}_{2,4}$. Lines and

represent the present semi-analytical results, and symbols $(*, \circ$, and $\square)$ denote the numerical results.

It can be seen from Figs. 3 and 4 that the present semi-analytical results of both added-mass and radiation damping coefficients are in excellent agreement with the numerical modelling results, which implies that the semi-analytical model proposed in this paper is correct.

It is also found that for the truncated cylinder with the cross section of a cosine radius function, $R(\theta)=0.5 h[1+0.1 \cos (3 \theta)], \quad F_{\mathrm{R}, 1}^{(1)}=F_{\mathrm{R}, 2}^{(2)}, \quad F_{\mathrm{R}, 4}^{(4)}=F_{\mathrm{R}, 5}^{(5)}, \quad F_{\mathrm{R}, 5}^{(1)}=-F_{\mathrm{R}, 4}^{(2)}$ are satisfied for the entire range of wave frequencies. Actually, these expressions are satisfied because of symmetry, as explained in Appendix B.

In addition to the cylinder with the cross section of a cosine radius function, a truncated circular cylinder studied analytically by Yeung (1981) is selected to validate the present semi-analytical model. As shown in Fig. 5, the present results of added-mass and radiation damping are in excellent agreement with those in Yeung (1981). 
(a)

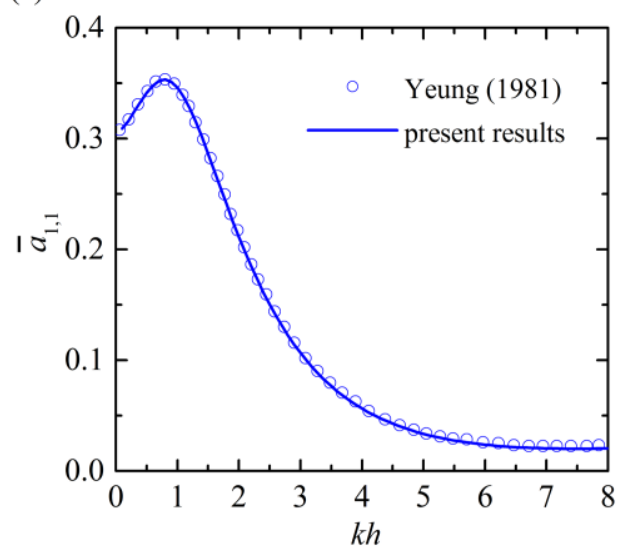

(c)

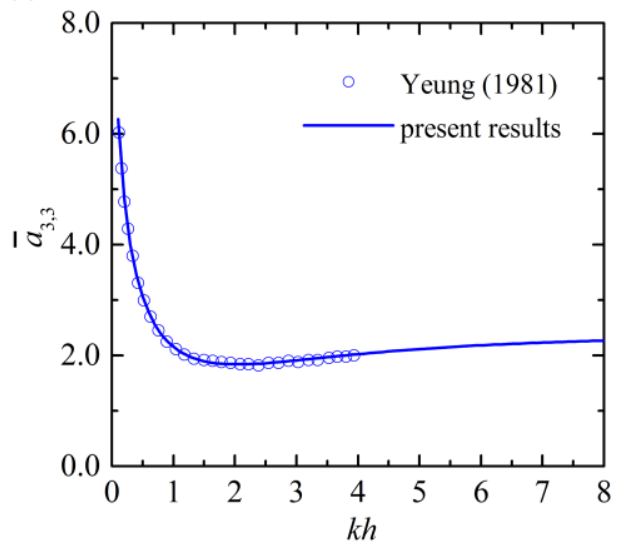

(e)

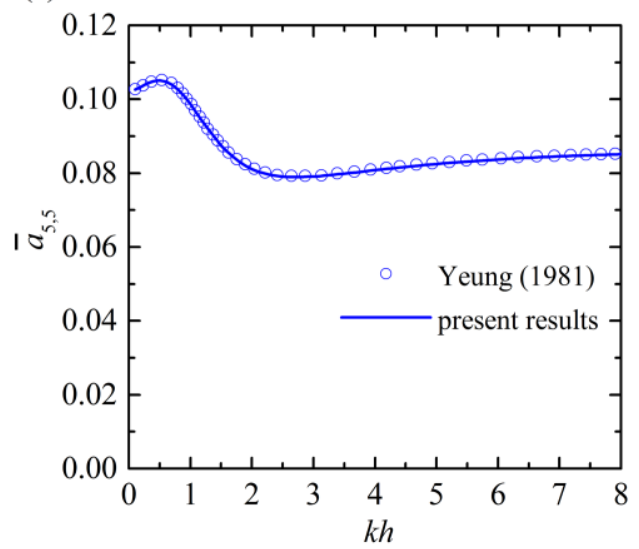

(b)

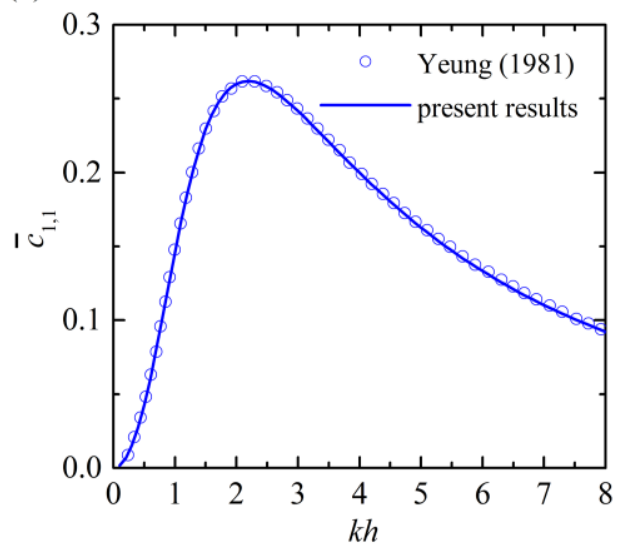

(d)

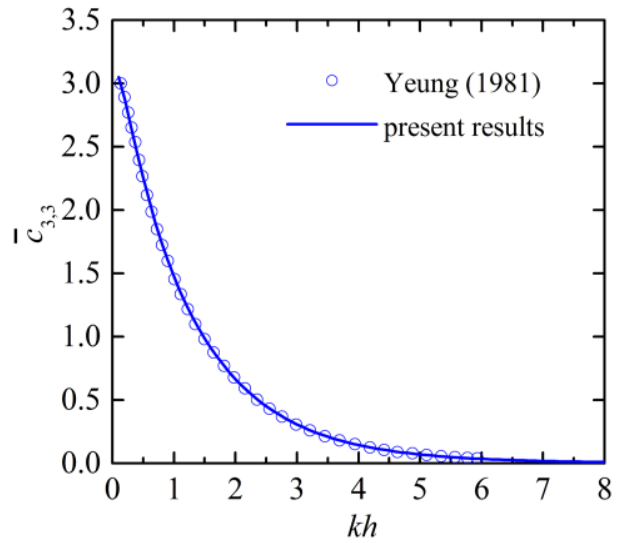

(f)

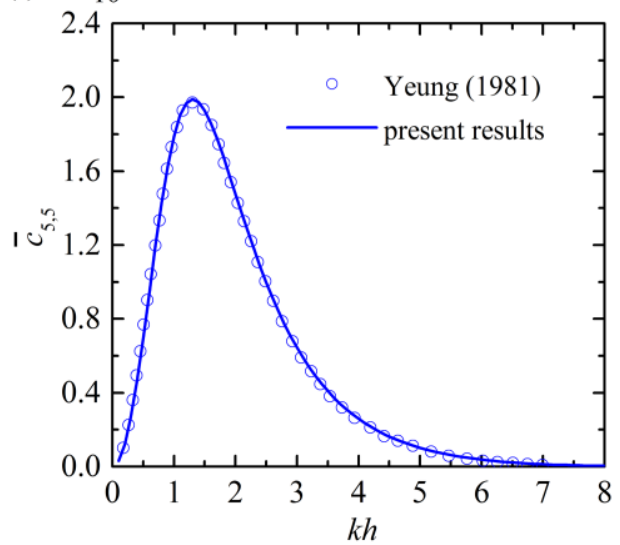

Fig. 5. Comparison of present semi-analytical results in terms of normalised added-mass and radiation damping with those in Yeung (1981) for a vertical circular cylinder, $R(\theta)=h, d=0.25 h$ : (a)

$$
\bar{a}_{1,1} ; \text { (b) } \bar{c}_{1,1} \text {; (c) } \bar{a}_{3,3} \text {; (d) } \bar{c}_{3,3} \text {; (e) } \bar{a}_{5,5} \text {; (f) } \bar{c}_{5,5} \text {. }
$$

\section{$5 \quad 5.2$ Additional case studies with different cross sections}

In this subsection, truncated cylinders with "circular", "cosine", "elliptical" and "quasi-elliptical" cross sections (Fig.6) are studied and compared with one another using the present validated semi-analytical model. 


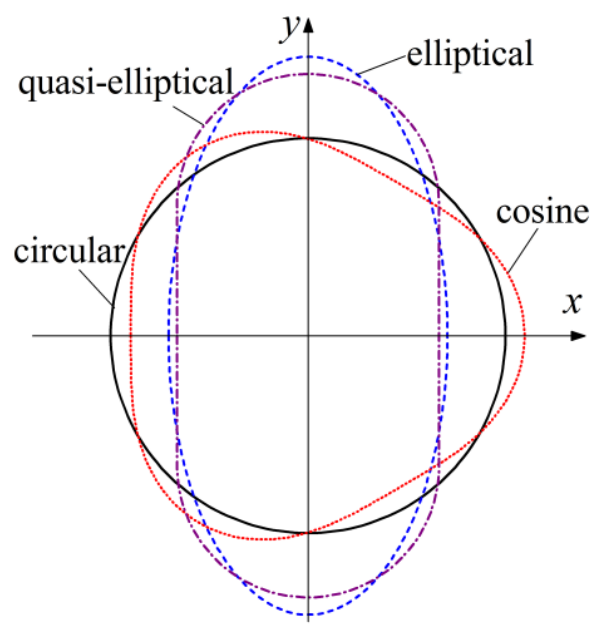

Fig. 6. Schematic of the truncated cylinder with 4 different cross sections

The exact radius function and the corresponding $\partial S / \partial \theta$ for these four cross sections are listed in Table 1. All these cross sections have the same value of area, i.e., $0.25 \pi h^{2}$. The axial ratio (i.e., the length in the $y$-axis direction relative to the length in the $x$-axis direction) for the cases of both "elliptical" and "quasi-elliptical" is 2.0.

Table 1 Radius function and $\frac{\partial S}{\partial \theta}$ of the cross section for the four case studies

\begin{tabular}{|c|c|c|}
\hline shape & $\frac{2 R(\theta)}{h}$ & $\frac{2 \partial S}{h \partial \theta}$ \\
\hline circular & 1 & 0 \\
\hline cosine & $\sqrt{\frac{1}{1.005}}[1+0.1 \cos (3 \theta)]$ & $0.3 \sqrt{\frac{1}{1.005}} \sin (3 \theta)$ \\
\hline elliptical & $\left(\frac{1}{2} \sin ^{2} \theta+2 \cos ^{2} \theta\right)^{-1 / 2}$ & $-\frac{3}{2}\left(\frac{1}{2} \sin ^{2} \theta+2 \cos ^{2} \theta\right)^{-3 / 2} \sin \theta \cos \theta$ \\
\hline $\begin{array}{c}\text { quasi-elliptic } \\
\text { al }\end{array}$ & $\left\{\begin{array}{l}\frac{\sqrt{\pi /(4+\pi)}}{\cos \theta}, \quad 0 \leq \theta \leq \frac{\pi}{4}, \frac{7 \pi}{4} \leq \theta \leq 2 \pi \\
\frac{-\sqrt{\pi /(4+\pi)}}{\cos \theta}, \quad \frac{3 \pi}{4} \leq \theta \leq \frac{5 \pi}{4} \\
2 \sqrt{\pi /(4+\pi)}|\sin \theta|, \frac{\pi}{4} \leq \theta \leq \frac{3 \pi}{4}, \frac{5 \pi}{4} \leq \theta \leq \frac{7 \pi}{4}\end{array}\right.$ & $\begin{cases}\frac{-\sqrt{\pi /(4+\pi)} \tan \theta}{\cos \theta}, & 0 \leq \theta \leq \frac{\pi}{4}, \frac{7 \pi}{4} \leq \theta \leq 2 \pi \\
\frac{\sqrt{\pi /(4+\pi)} \tan \theta}{\cos \theta}, & \frac{3 \pi}{4} \leq \theta \leq \frac{5 \pi}{4} \\
-2 \sqrt{\pi /(4+\pi)} \cos \theta, & \frac{\pi}{4} \leq \theta \leq \frac{3 \pi}{4} \\
2 \sqrt{\pi /(4+\pi)} \cos \theta, & \frac{5 \pi}{4} \leq \theta \leq \frac{7 \pi}{4}\end{cases}$ \\
\hline
\end{tabular}

Figures 7 and 8 show the semi-analytical results of added-mass and radiation damping coefficients, respectively. 
(a)

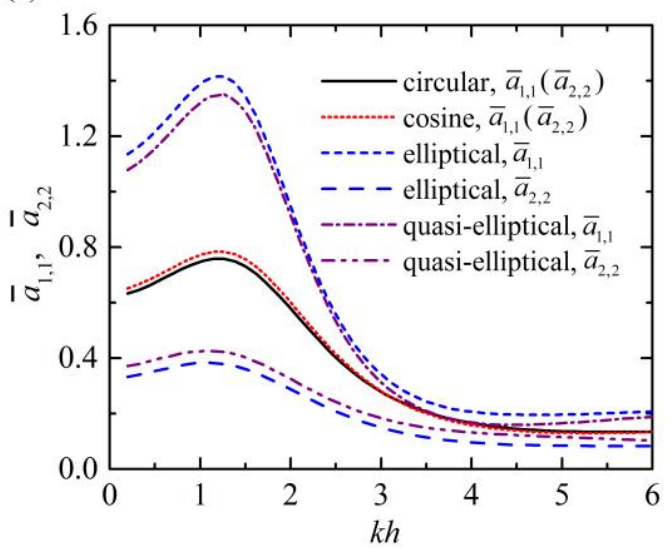

(c) $\times 10^{-1}$

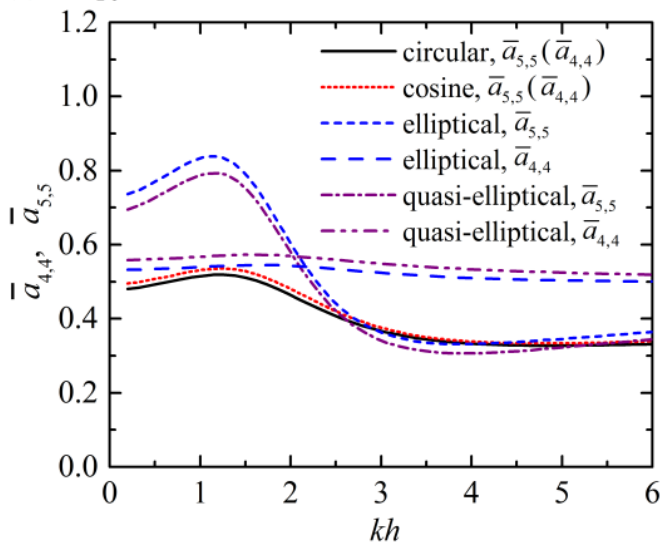

(b)

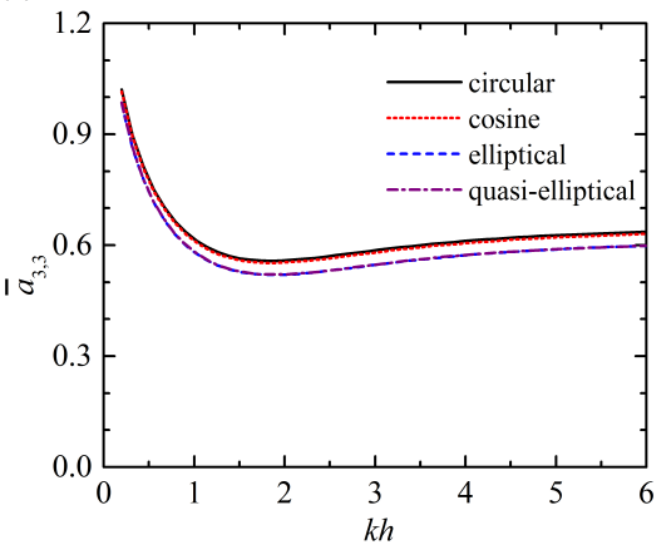

(d) $\times 10^{-1}$

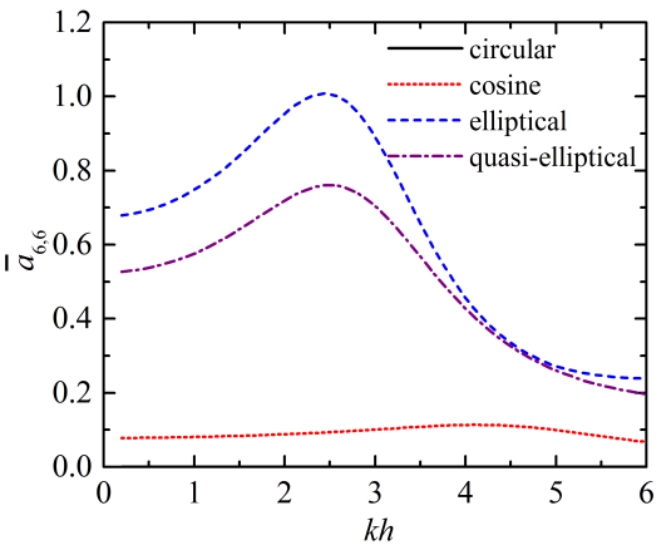

(e)

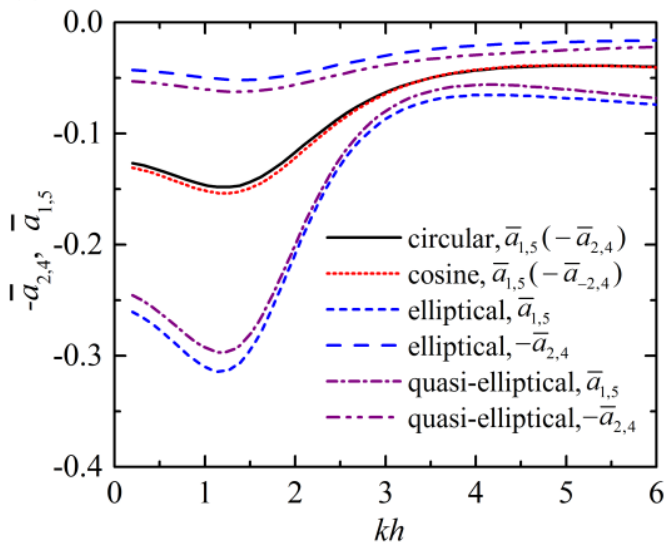


(a)

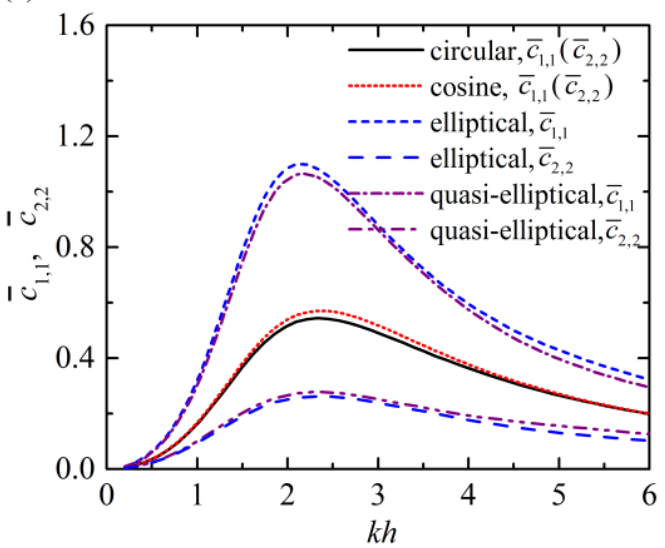

(c) $\times 10^{-2}$

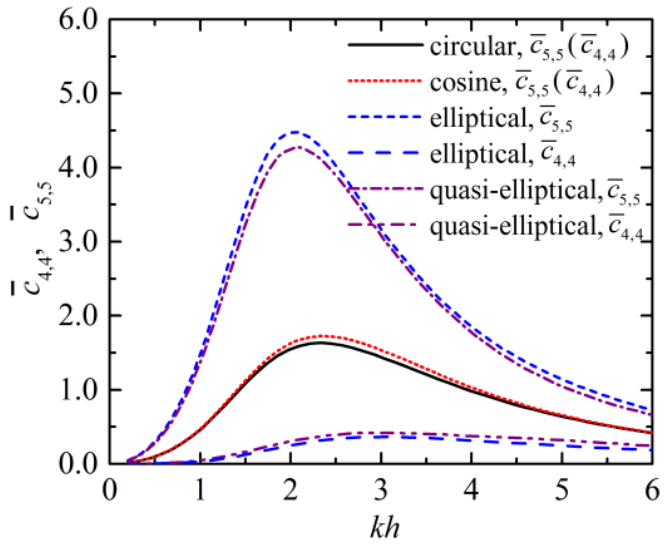

(b)

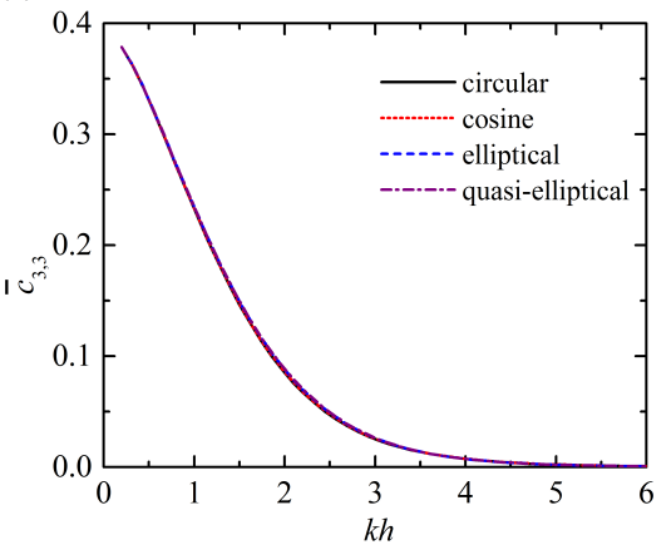

(d) $\times 10^{-2}$

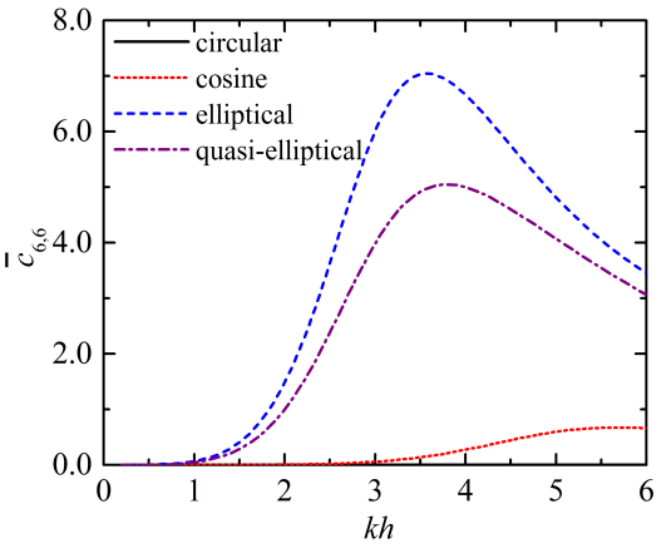

(e)

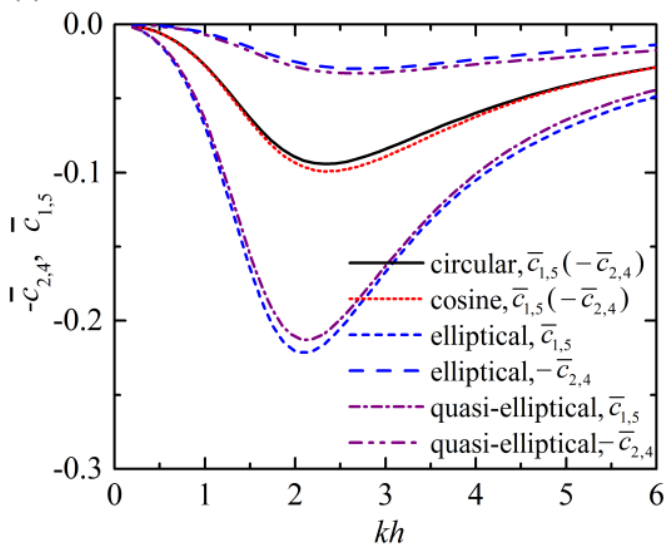

Fig. 8. Normalised radiation damping vs. $k h$ for the truncated cylinder with 4 different cross sections, $d / h=0.5$ : (a) $\bar{c}_{1,1}$ and $\bar{c}_{2,2}$; (b) $\bar{c}_{3,3}$; (c) $\bar{c}_{4,4}$ and $\bar{c}_{5,5}$; (d) $\bar{c}_{6,6}$; (e) $\bar{c}_{1,5}$ and $\bar{c}_{2,4}$.

It may be inferred from Figs. 7 and 8 that all of the normalised added-mass values for the different cross sections corresponding to the same mode have similar variation trends against $k h$, as well as normalised radiation damping. Since the "circular" and "cosine" cross sections as shown in Fig. 6 are close to each other, the corresponding values of these two truncated cylinders are not very different from each other. And the same may be said of the truncated cylinders with "elliptical" and "quasi-elliptical" cross sections. As illustrated in Fig. 7a, for $k h<3.0$, the longer the truncated cylinder in the $y$-direction, the larger the value of the dimensionless added-mass in surge mode due to the surge oscillation $\left(\bar{a}_{1,1}\right)$. This applies also to the dimensionless radiation 
1 damping in surge mode due to the surge oscillation $\left(\bar{c}_{1,1}\right)$, even for the entire range of $k h$ studied

2 (Fig. 8a). Even though the length in the $x$-direction of the cylinder with "cosine" cross section is slightly smaller than that with "circular" shape, slightly larger values of its corresponding $\bar{a}_{2,2}$

4 and $\bar{c}_{2,2}$ are obtained compared with those of "circular" shape for $k h<3.0$ and the whole range of

$5 \quad k h$ studied, respectively. The values of $\bar{a}_{3,3}$ and $\bar{c}_{3,3}$ of different cylinders with the same cross 6 section area, especially the latter, are found very close to each other regardless of the cylinder 7 section shape (Figs.7b and 8b). Following the analysis of Yeung (1981), for all the four cylinders with different shapes of cross section, it might be learnt from Figs.7b and $8 \mathrm{~b}$ that $\bar{c}_{3,3}$ decays

9 monotonically from a finite value as $k h$ increases, whereas $\bar{a}_{3,3}$ starts from a logarithmic behaviour and attains a minimum before approaching its asymptotic infinite-frequency limit. In the case of the "elliptical" and "quasi-elliptical" cross sections, the values of $\bar{a}_{4,4}$ are less affected by the relative water depth $(k h)$ than they are in the case of the other cross sections (Fig. 7c). Variations of $\bar{c}_{4,4}$ and $\bar{c}_{5,5}$ with $k h$ for different cylinders as given in Fig. $8 \mathrm{c}$ are found to be very similar to those of $\bar{c}_{1,1}$ and $\bar{c}_{2,2}$ (Fig. 8a). For the "circular" cross section, we have $\partial S / \partial \theta=0$, thus there are no radiation forces due to its yaw motion with the inviscid water assumption inherent in potential flow theory, resulting in $\bar{a}_{6,6}=\bar{c}_{6,6}=0$, as may be seen in Figs. $7 \mathrm{~d}$ and $8 \mathrm{~d}$. The largest values of $\bar{a}_{6,6}$ for the "elliptical" and "quasi-elliptical" cross sections are 0.101 and 0.076 , respectively, both occurring at $k h=2.45$. As a comparison, the peak value of $\bar{a}_{6,6}$ for the "cosine" cross section is a mere 0.011 , at $k h=4.11$. The corresponding largest values of $\bar{c}_{6,6}$ for "cosine", "elliptical" and "quasi-elliptical" cross sections are 0.007, 0.070 and 0.050, occurring at $k h=5.64,3.51$ and 3.75 , respectively (Fig. $8 \mathrm{~d}$ ).

Figures $7 \mathrm{e}$ and $8 \mathrm{e}$ present the variation of $\bar{a}_{1,5}, \bar{a}_{2,4}$ and $\bar{c}_{1,5}, \bar{c}_{2,4}$, separately. It can be seen that the values of these hydrodynamic coefficients for the "quasi-elliptical" cross section are closer to those of "elliptical" cross section than they are to those of "circular" and "cosine" sections.

\subsection{Effect of cylinder draft}

As mentioned by Chakrabarti et al. (2006), there are several challenges in the design, construction and installation of a floating caisson to be used as a bridge pier, not least the fact that the wave induced hydrodynamic forces vary significantly during installation. In this section, the 
1 effect of the cylinder draft on hydrodynamic coefficients of the cylinder of "quasi-elliptical" cross 2 section is investigated. The radius function and the corresponding $\partial S / \partial \theta$ for the "quasi-elliptical" 3 case as listed in Table 1 in Section 5.2 are adopted. Semi-analytical values for the dimensionless 4 added-mass and radiation damping coefficients for five different cases with the cylinder draft 5 ranging from $d / h=0.1$ to $d / h=0.9$ are presented in Figs. 9 and 10, respectively.

(a)

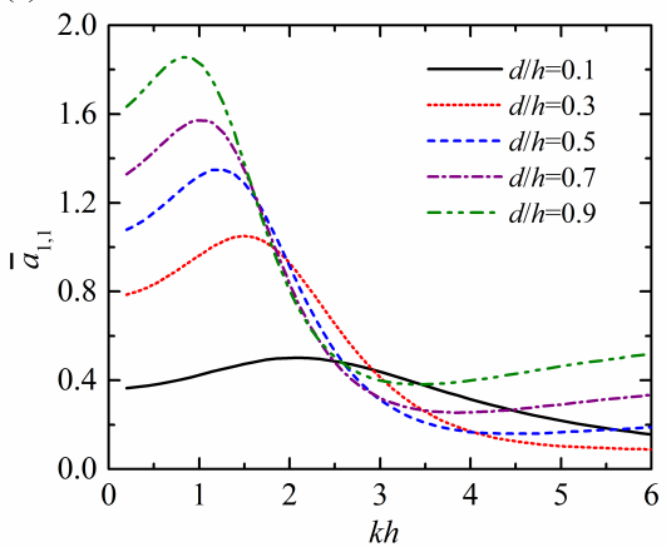

(c)

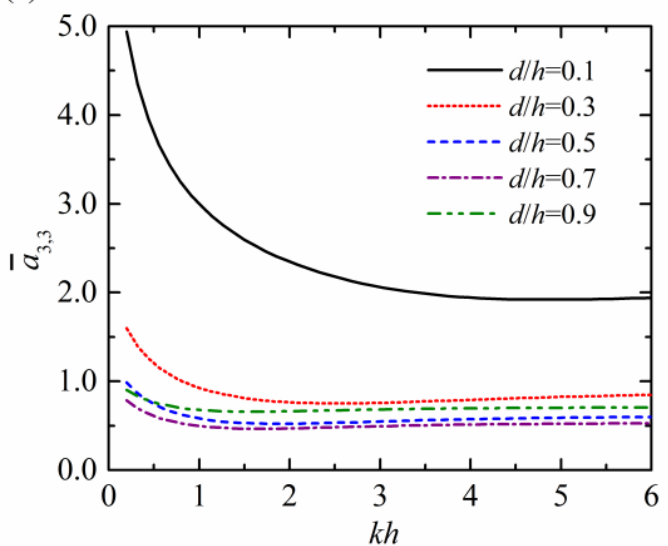

(e)

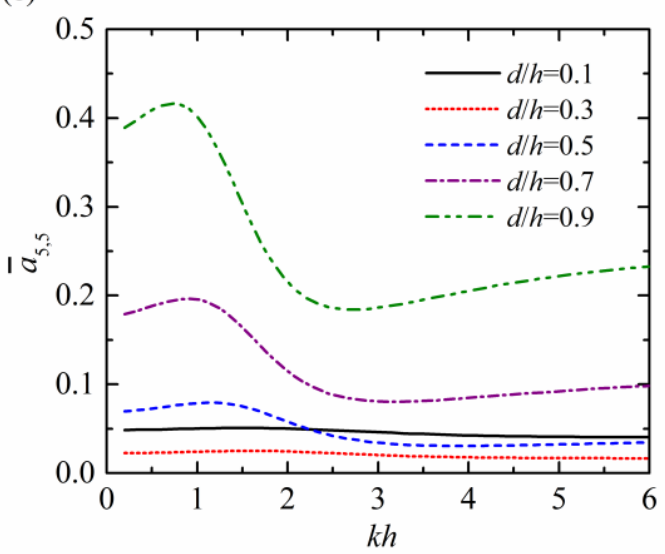

(b)

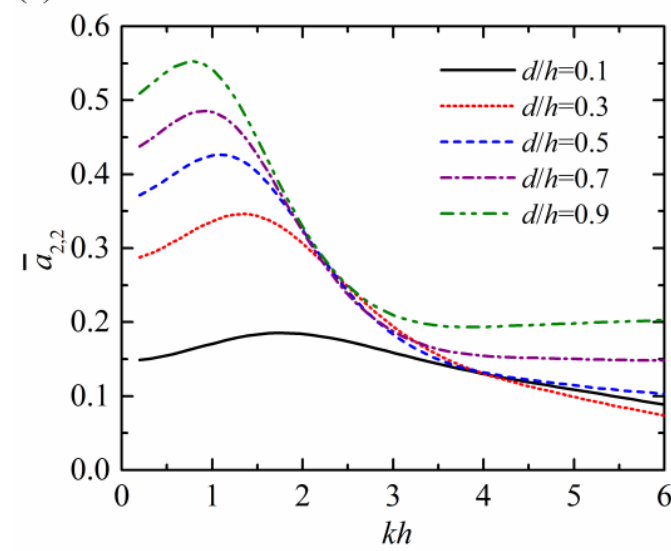

(d)

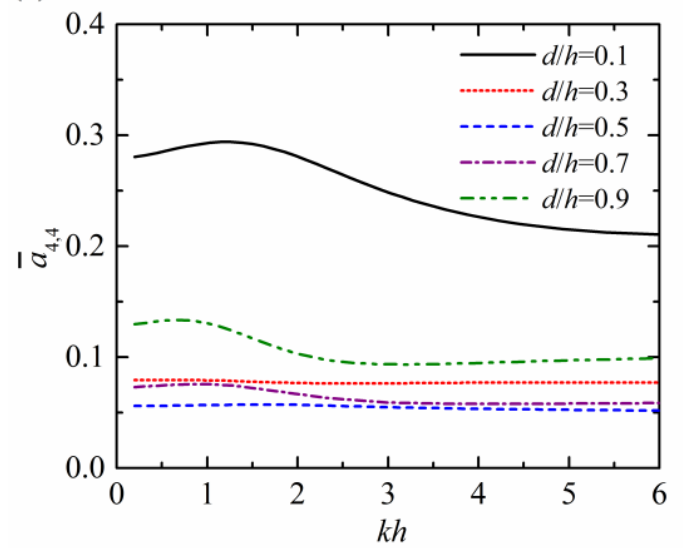

(f) $\times 10^{-1}$

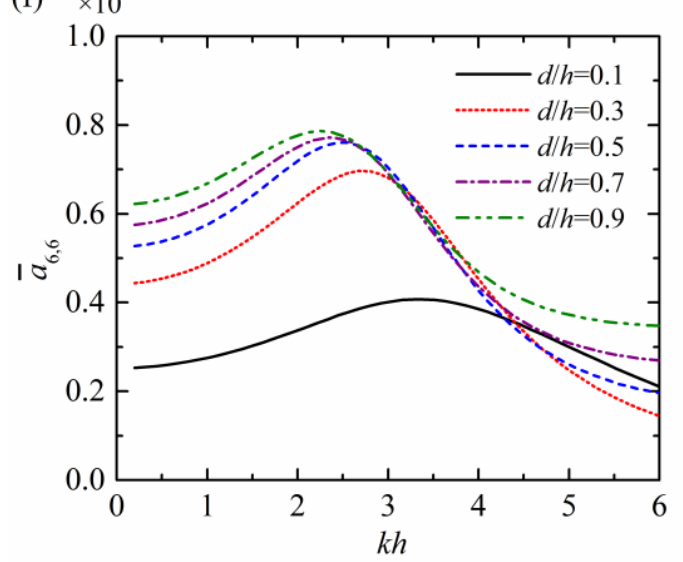


(g)

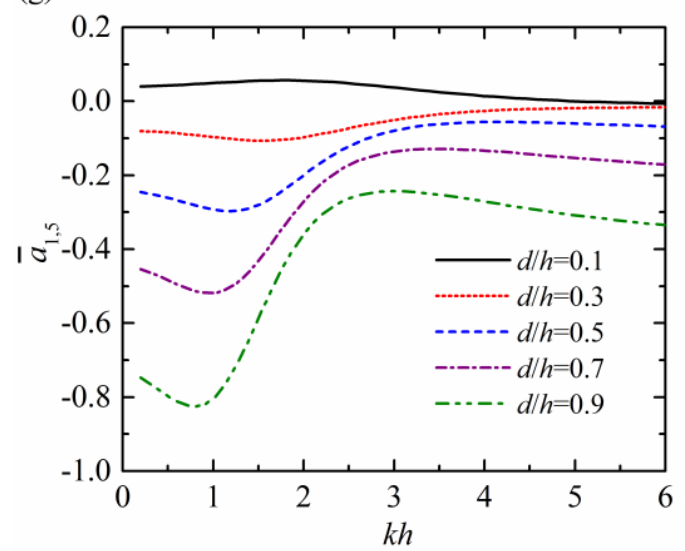

(h)

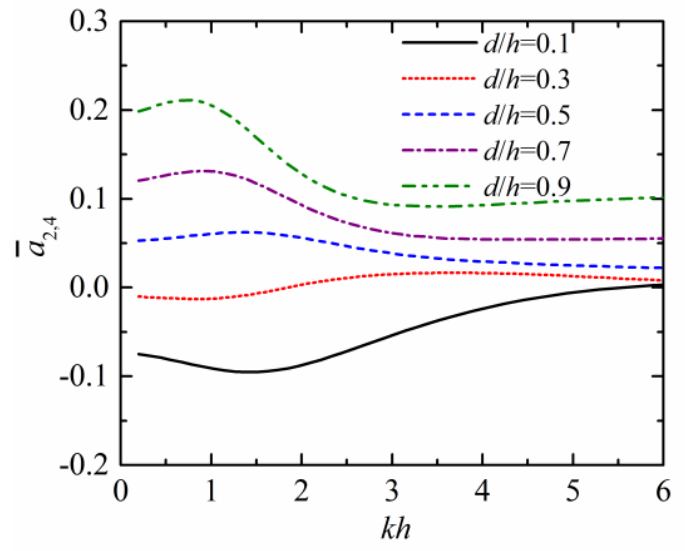

2

3

4

(a)

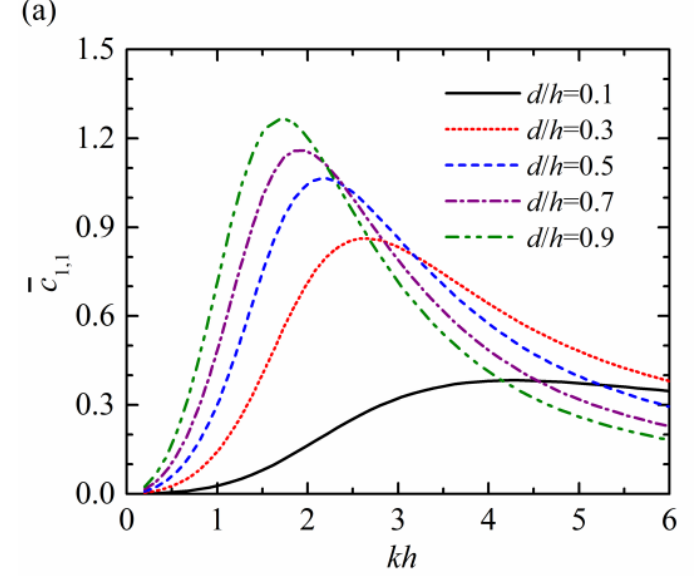

(c)

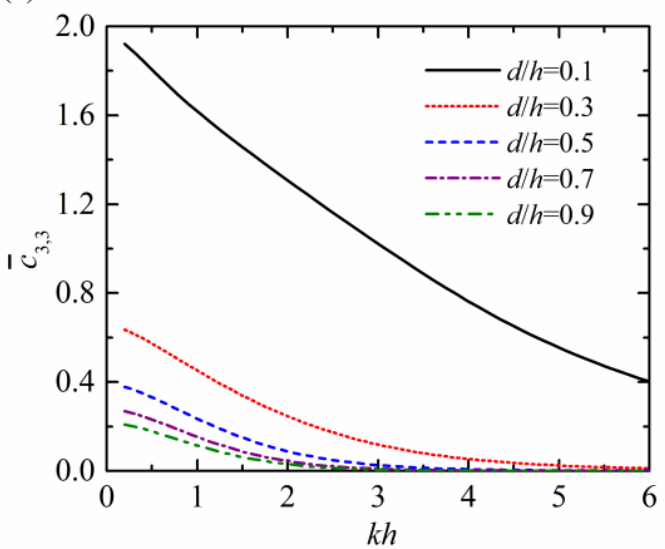
with different draft: (a) $\bar{a}_{1,1}$; (b) $\bar{a}_{2,2}$; (c) $\bar{a}_{3,3}$; (d) (b)

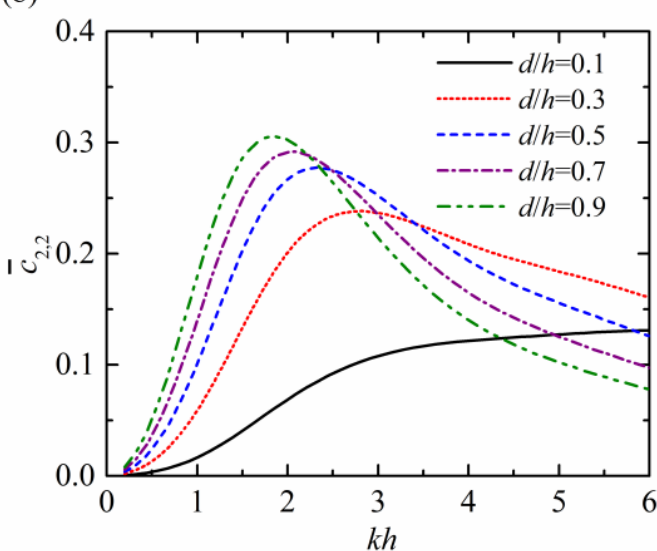

(d)

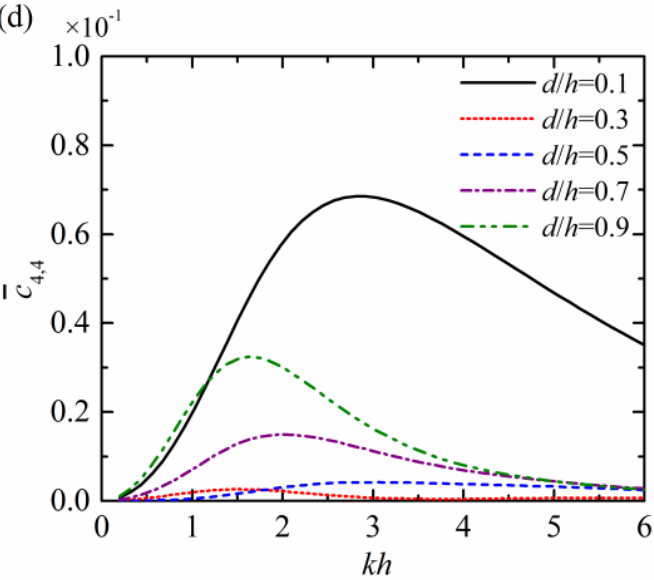

Fig. 9. Normalised added-mass vs $k h$ for the truncated cylinder of "quasi-elliptical" cross section

(d) $\bar{a}_{4,4} ;(\mathrm{e}) \bar{a}_{5,5} ;$ (f) $\bar{a}_{6,6} ;$ (g) $\bar{a}_{1,5} ;$ (h) $\bar{a}_{2,4}$.

\section{5}


(e) $\times 10^{-1}$

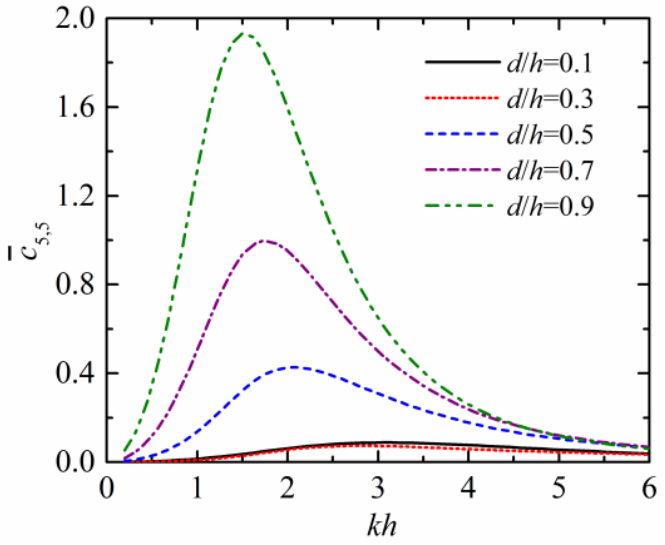

(g)

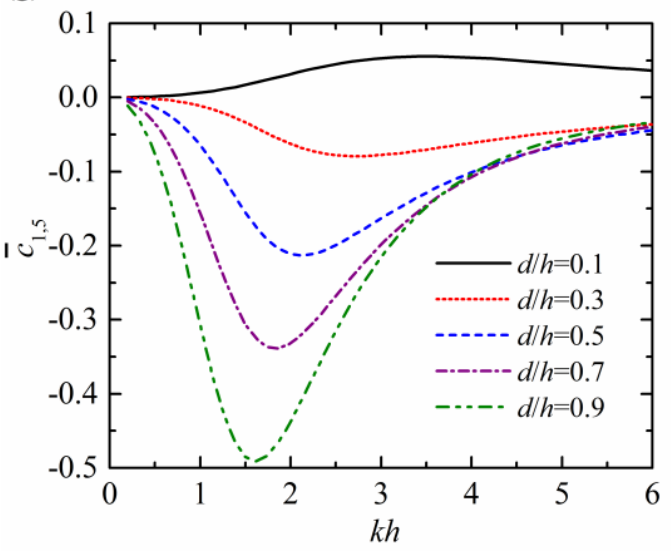

(f) $\times 10^{-2}$

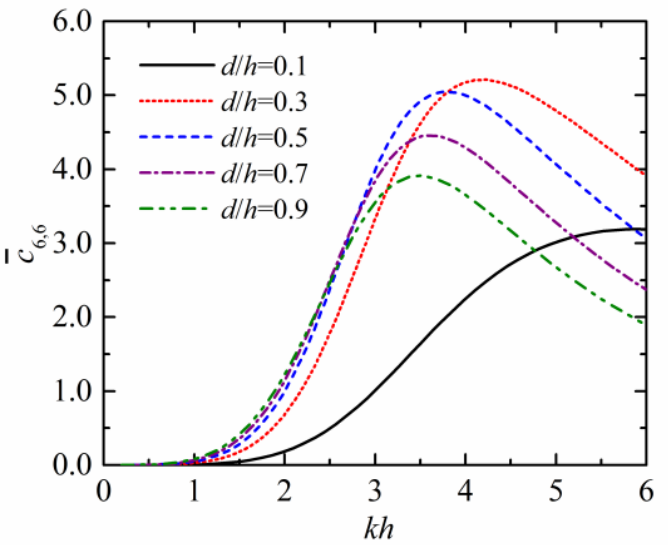

(h) $\times 10^{-1}$

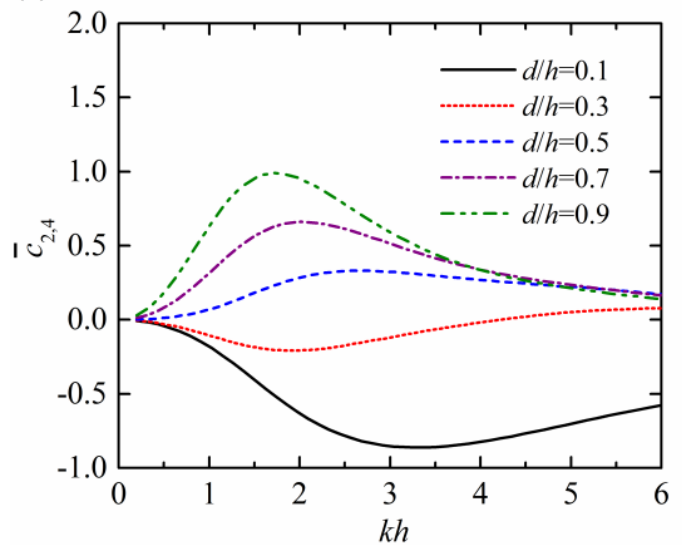

3

4

5

6

7

Fig. 10. Normalised radiation damping vs $k h$ for the truncated cylinder of "quasi-elliptical" cross section with different draft: (a) $\bar{c}_{1,1}$; (b) $\bar{c}_{2,2}$; (c) $\bar{c}_{3,3} ;$ (d) $\bar{c}_{4,4}$; (e) $\bar{c}_{5,5}$; (f) $\bar{c}_{6,6} ;$ (g) $\bar{c}_{1,5}$; (h) $\bar{c}_{2,4}$.

For the cylinder of "quasi-elliptical" cross section oscillating in long waves, $k h<1.0$, as shown in Figs. 9a and 9b, the larger the draft is, the larger the values of both $\bar{a}_{1,1}$ and $\bar{a}_{2,2}$ of the cylinder are. For $k h$ ranging from 0.5 to 3.0 , there are peaks for both $\bar{a}_{1,1}-k h$ and $\bar{a}_{2,2}-k h$ curves. The cylinder with a larger draft has larger peak values of $\bar{a}_{1,1}$ and $\bar{a}_{2,2}$, whereas has smaller corresponding $k h$ where the peak occurs. Similar effect of the draft can also be found in Figs. 10a and 10b on $\bar{c}_{1,1}$ and $\bar{c}_{2,2}$, except for the case of $d / h=0.1$, as shown in Fig. 10b, that $\bar{c}_{2,2}$ increases all the time with the increases of $k h$ and there is no peak of the $\bar{c}_{2,2}-k h$ curve for all the wave number considered. It can be learnt from Fig. $9 \mathrm{c}$ that as $d / h$ increases from 0.1 to 0.7 , $\bar{a}_{3,3}$ turns smaller and smaller independently with $k h$. While if $d / h$ keeps increasing, i.e. from 0.7 to $0.9, \bar{a}_{3,3}$ will otherwise increase. When $k h \rightarrow \infty, \bar{a}_{3,3}$ tends to be a finite constant. As a 
comparison, as given in Fig. 10c, $\bar{c}_{3,3}$ decreases all the time with $d / h$ increasing from 0.1 to 0.9 . For $k h \rightarrow 0$, there is an inversely-proportional relationship between $\bar{c}_{3,3}$ and $d / h$. Actually, for $k h$ $\rightarrow 0, \bar{c}_{3,3} \rightarrow \pi h /(16 d)$ is satisfied, which can be proved with the assumption of small-body approximation and the reciprocity relation between heave radiation damping and heave excitation force (Falnes, 2002). As presented in Fig. 9d, for arbitrary $k h, \bar{a}_{4,4}$ decreases with increasing $d / h$ ranging from 0.1 to 0.5 , while it increases as $d / h$ increases from 0.5 to 0.9 . As a comparison, $\bar{c}_{4,4}$, $\bar{a}_{5,5}$ and $\bar{c}_{5,5}$ also first decrease with increasing draft, then increase, whereas the corresponding $d / h$ where the minimum $\bar{c}_{4,4}, \bar{a}_{5,5}$ and $\bar{c}_{5,5}$ occur are smaller than those of $\bar{a}_{4,4}$, as illustrated in Figs. 10d, 9e and 10e. The influence law of cylinder draft on the wave radiation moments $F_{\mathrm{R}, i}^{(i)}$ $(i=4,5)$ in terms of $\bar{a}_{4,4}, \bar{c}_{4,4}, \bar{a}_{5,5}$ and $\bar{c}_{5,5}$ can be explained from the view of the constitution of $F_{\mathrm{R}, i}^{(i)}(i=4,5)$. When $d / h$ is very small, extremely when $d / h \rightarrow 0, \quad F_{\mathrm{R}, i}^{(i)} \quad(i=4,5)$ will not be vanishing and it will be dominated by the pressure at the bottom of the cylinder, therefore the dimensionless quantities of the corresponding hydrodynamic coefficients could be very large from Eq. (49). As $d / h$ increases from the small value, e.g. $d / h=0.1$, according to Eq. (49), $\bar{a}_{4,4}$, $\bar{c}_{4,4}, \bar{a}_{5,5}$ and $\bar{c}_{5,5}$ will apparantly decrease first when the contribution of the pressure on side wall still makes up a small proportion of $F_{\mathrm{R}, i}^{(i)}(i=4,5)$. While after $d / h$ being increased to some extent that the pressure loading on the side wall of the cylinder dominates $F_{\mathrm{R}, i}^{(i)}(i=4,5), F_{\mathrm{R}, i}^{(i)}$ will shows an approximate exponential growth with the further increase of $d / h$, leading to an increasing trend of $\bar{a}_{4,4}, \bar{c}_{4,4}, \bar{a}_{5,5}$ and $\bar{c}_{5,5}$. Variations of $\bar{a}_{6,6}$ with $k h$ for different draft as given in Fig. 9f are very similar to those of $\bar{c}_{1,1}$ as illustrated in Fig. 10a. The peak value of $\bar{a}_{6,6}$ for $d / h=0.9$ is 0.079 occurring at $k h=2.21$, larger than the peak value for any other cylinder with smaller draft. It can be seen from Fig. $10 \mathrm{f}$ that $\bar{c}_{6,6}$ first increases with increasing $k h$, then decreases, leading to peaks for $\bar{c}_{6,6}-k h$ curves, except for the case of $d / h=0.1$, which increases all the time with increasing $k h$. The largest peak value of the $\bar{c}_{6,6}-k h$ curves is 0.0521 , occurring at $k h=4.22$ for the cylinder with $d / h=0.3$. As exhibited in Figs. $9 \mathrm{~g}$ and $10 \mathrm{~g}, \bar{a}_{1,5}$ and $\bar{c}_{1,5}$ decrease 
all the time with increasing $d / h$ for the whole range of $k h$, except for $\bar{c}_{1,5}$ when $k h>3.5$. As a comparison of the effects on $\bar{a}_{1,5}$ and $\bar{c}_{1,5}$, on the contrary, $d / h$ shows an opposite effect on $\bar{a}_{2,4}$ and $\bar{c}_{2,4}$ (Figs. 9h and 10h).

\section{Conclusions}

In this paper, a semi-analytical model based on linear potential flow theory is established to solve the radiation problem from a truncated cylinder of arbitrary cross section floating in finite water depth. The water domain is divided into an interior domain beneath the cylinder and an exterior domain outside the cylinder column. The method of separation of variables is used to express the radiated potential in both domains. The terms associated to the radius function at the interface of the two domains (continuity conditions) are dealt with Fourier series expansion method. On this basis the eigen-function matching method is applied to determine the unknown coefficients in the expressions of radiated potential.

The semi-analytical model thus developed is validated by comparing its results of added-mass and radiation damping for a cylinder of cosine cross section with numerical modelling results. Excellent agreement is obtained between the semi-analytical and numerical modelling results. In addition, a truncated circular cylinder that has been studied by other researchers is taken as another case to validate the present semi-analytical model. Once validated, the semi-analytical model is applied to obtain the hydrodynamic coefficients for cylinders with four different cross sections, i.e. "circular", "cosine", "elliptical" and "quasi-elliptical", with the same cross section area. Additionally, the influence of the draft on the wave radiation from the truncated cylinder of "quasi-elliptical" cross section- of particular interest for engineers installing a floating caisson to be used as a bridge pier - is investigated.

The semi-analytical results of these cases reveal that:

(a) For a given cross-section area, the dimensionless added-mass and radiation damping in heave mode due to the heave oscillation $\left(\bar{a}_{3,3}\right.$ and $\left.\bar{c}_{3,3}\right)$, especially the latter, of cylinders with the same cross section area and draft are found to be very close to each other regardless of the cross section shape.

(b) For a given cross-section area and draft, the largest values of the dimensionless added-mass and radiation damping in yaw mode due to the yaw oscillation of the cylinder $\left(\bar{a}_{6,6}\right.$ and $\bar{c}_{6,6}$ ) for the elliptical cross sections are 0.101 and 0.070 , respectively, far greater than for “quasi-elliptical" (0.076 and 0.050$)$ and "cosine" (0.011 and 0.007) cross sections.

(c) For the cylinder of "quasi-elliptical" cross section, when the relative water depth tends to zero $(k h \rightarrow 0)$, there is an inversely proportional relationship between $\bar{c}_{3,3}$ and the relative cylinder draft $(d / h)$.

(d) For the cylinder of "quasi-elliptical" cross section at any specified $k h$, as $d / h$ increases from 0.1 to 0.9 , the values of the dimensionless added-mass and radiation damping in roll/pitch 
mode due to the roll/pitch oscillation of the cylinder $\left(\bar{a}_{4,4}, \bar{c}_{4,4}, \bar{a}_{5,5}\right.$ and $\left.\bar{c}_{5,5}\right)$ first decrease and then increase, resulting in minimal values with suitable selections of $d / h$.

The present work was concerned with the hydrodynamic problem of one vertical cylinder with arbitrary cross sections. If several cylinders are deployed in proximity, the hydrodynamic interactions between them may be relevant. Wave diffraction and radiation from multiple truncated cylinders of arbitrary cross section will be the object of future work.

Acknowledgements

The research was supported by China Postdoctoral Science Foundation (Grant No. 2016M601041, 2017T100085), National Natural Science Foundation of China (51679124, 51479092) and Intelligent Community Energy (ICE), INTERREG V FCE, European Commission (Contract No. 5025). The corresponding author wishes to thank PhD candidate Jiabin Liu from Harbin Institute of Technology for valuable discussions.

Appendix. A Derivation process of the formulas and calculation for $A_{m, l}^{(i)}$ and $B_{m, l}^{(i)}$

$$
\text { After multiplying both sides of Eq. (32) by } \frac{\cos \left[\beta_{\varsigma}(z+h)\right] \mathrm{e}^{-\mathrm{i} \tau \theta}}{2 \pi(h-d)} \text { and integrating for } z \in[-h \text {, }
$$

$-d]$ and $\theta \in[0,2 \pi]$, we get, for any pair of integer $(\tau, \zeta)$ :

$$
-\sum_{m=-\infty}^{\infty} \Lambda_{m, \tau, \varsigma}^{(1)} A_{m, \varsigma}^{(i)}+\sum_{m=-\infty}^{\infty} \sum_{l=0}^{\infty} B_{m, l}^{(i)} L_{l, \zeta} \Lambda_{m, \tau, l}^{(2)}=f_{\tau, \varsigma}^{(1), i},
$$

where

$$
\begin{aligned}
\Lambda_{m, \tau, \varsigma}^{(1)}= & \left\{\begin{array}{ll}
f_{m, \varsigma, \tau-m}^{R}, \quad \varsigma=0 \\
\frac{1}{2} \tilde{f}_{m, \varsigma, \tau-m}^{I}, \quad \varsigma=1,2,3, \ldots
\end{array} ; \Lambda_{m, \tau, l}^{(2)}=\left\{\begin{array}{ll}
f_{m, 0, \tau-m}^{H}, & l=0 \\
f_{m, l, \tau-m}^{K}, & l=1,2,3, \ldots
\end{array},\right.\right. \\
& L_{l, \varsigma}=\frac{1}{h-d} \int_{-h}^{-d} \frac{Z_{l}(z) \cos \left[\beta_{\varsigma}(z+h)\right]}{Z_{l}(0)} \mathrm{d} z \\
= & \begin{cases}\frac{(-1)^{\varsigma}(h-d) k_{0} \sinh \left[k_{0}(h-d)\right]}{\left[(h-d)^{2} k_{0}^{2}+\varsigma^{2} \pi^{2}\right] \cosh \left(k_{0} h\right)}, & l=0 ; \varsigma=0,1,2 \ldots, \\
\frac{(-1)^{\varsigma}(h-d) k_{l} \sin \left[k_{l}(h-d)\right]}{\left[(h-d)^{2} k_{l}^{2}-\varsigma^{2} \pi^{2}\right] \cos \left(k_{l} h\right)}, & l=1,2,3 \cdots ; \varsigma=0,1,2 \ldots\end{cases}
\end{aligned}
$$




$$
f_{\tau, \varsigma}^{(1), i}=\left\{\begin{array}{lr}
0, & i=1,2,6 \\
\frac{1}{4(h-d)^{2}}\left[2 \delta_{\tau, 0} I Z_{\varsigma}-\delta_{\zeta, 0}(h-d) f_{2,0, \tau}^{R}\right], & i=3 \\
\frac{1}{16 \mathrm{i}(h-d)^{2}}\left[4 I Z_{\zeta}\left(f_{1,0, \tau-1}^{R}-f_{1,0, \tau+1}^{R}\right)-\delta_{\zeta, 0}(h-d)\left(f_{3,0, \tau-1}^{R}-f_{3,0, \tau+1}^{R}\right)\right], & i=4, \\
\frac{-1}{16(h-d)^{2}}\left[4 I Z_{\zeta}\left(f_{1,0, \tau-1}^{R}+f_{1,0, \tau+1}^{R}\right)-\delta_{\zeta, 0}(h-d)\left(f_{3,0, \tau-1}^{R}+f_{3,0, \tau+1}^{R}\right)\right], & i=5
\end{array}\right.
$$

2 in which

3

$$
I Z_{\zeta}=\int_{-h}^{d} \cos \left[\beta_{\varsigma}(z+h)\right](z+h)^{2} \mathrm{~d} z= \begin{cases}\frac{(h-d)^{3}}{3}, & \varsigma=0 \\ \frac{2(-1)^{\varsigma}(h-d)^{3}}{\varsigma^{2} \pi^{2}}, & \varsigma=1,2,3, \ldots\end{cases}
$$

4 Similarly, by multiplying both sides of Eq. (33) with $\frac{Z_{\varsigma}(z) \mathrm{e}^{-\mathrm{i} \tau \theta}}{2 \pi h}$ and integrating in $z \in[-h$,

$0]$ and $\theta \in[0,2 \pi]$, we get, for any pair of integer $(\tau, \zeta)$ :

6

$$
-\frac{h-d}{h} Z_{\varsigma}(0) \sum_{m=-\infty}^{\infty} \sum_{l=0}^{\infty} A_{m, l}^{(i)} L_{\varsigma, l} \Lambda_{m, \tau, l}^{(3)}+\sum_{m=-\infty}^{\infty} B_{m, \varsigma}^{(i)} \Lambda_{m, \tau, \varsigma}^{(4)}=f_{\tau, \varsigma}^{(2), i},
$$

7 where

8

$$
\Lambda_{m, \tau, l}^{(3)}=\left\{\begin{array}{ll}
\tilde{f}_{m, 0, \tau-m}^{R}, & l=0 \\
\tilde{f}_{m, l, \tau-m}^{I^{\prime}}, & l=1,2,3, \ldots
\end{array} \quad ; \quad \Lambda_{m, \tau, \varsigma}^{(4)}=\left\{\begin{array}{l}
\frac{f_{m, 0, \tau-m}^{H^{\prime}},}{Z_{0}(0)} \quad \varsigma=0 \\
\frac{f_{m, \varsigma, \tau-m}^{K^{\prime}},}{Z_{\varsigma}(0)} \quad \varsigma=1,2,3, \ldots
\end{array},\right.\right.
$$

9

$$
f_{\tau, \varsigma}^{(2), i}= \begin{cases}I_{\varsigma}^{(3)} f_{\tau}^{(5)}, & i=1 \\ I_{\varsigma}^{(3)} f_{\tau}^{(4)}, & i=2 \\ -\frac{I_{\varsigma}^{(1)}}{2(h-d)} f_{3,0, \tau}^{R}, & i=3 \\ -\left(I_{\varsigma}^{(4)}-z_{0} I_{\varsigma}^{(3)}\right) f_{\tau}^{(4)}+\frac{I_{\varsigma}^{(2)} f_{\tau}^{(4)}}{2(h-d)}-\frac{I_{\varsigma}^{(1)} f_{\tau}^{4^{\prime}}}{8(h-d)}, & i=4 \\ \left(I_{\varsigma}^{(4)}-z_{0} I_{\varsigma}^{(3)}\right) f_{\tau}^{(5)}-\frac{I_{\varsigma}^{(2)} f_{\tau}^{(5)}}{2(h-d)}+\frac{I_{\varsigma}^{(1)} f_{\tau}^{5^{\prime}}}{8(h-d)}, & i=5 \\ I_{\varsigma}^{(3)} f_{\tau}^{6^{\prime}}, & i=6\end{cases}
$$

10

in which

$$
I_{\zeta}^{(1)}=\int_{-h}^{-d} \frac{Z_{\zeta}(z)}{h} \mathrm{~d} z=\left\{\begin{array}{ll}
\frac{N_{0}^{-0.5} \sinh \left[k_{0}(h-d)\right]}{k_{0} h}, & \varsigma=0 \\
\frac{N_{\zeta}^{-0.5} \sin \left[k_{\zeta}(h-d)\right]}{k_{\varsigma} h}, & \varsigma=1,2,3, \ldots
\end{array},\right.
$$


1

$$
\begin{aligned}
& I_{\zeta}^{(2)}=\int_{-h}^{-d} \frac{Z_{\zeta}(z)}{h}(z+h)^{2} \mathrm{~d} z \\
& =\left\{\begin{array}{l}
\frac{N_{0}^{-0.5}}{k_{0}^{3} h}\left\{k_{0}^{2}(h-d)^{2} \sinh \left[k_{0}(h-d)\right]-2 k_{0}(h-d) \cosh \left[k_{0}(h-d)\right]+2 \sinh \left[k_{0}(h-d)\right]\right\}, \quad \varsigma=0 \\
\frac{N_{\zeta}^{-0.5}}{k_{\varsigma}^{3} h}\left\{k_{\varsigma}^{2}(h-d)^{2} \sin \left[k_{\varsigma}(h-d)\right]+2 k_{\varsigma}(h-d) \cos \left[k_{\varsigma}(h-d)\right]-2 \sin \left[k_{\varsigma}(h-d)\right]\right\}, \quad \varsigma=1,2,3, \ldots
\end{array}\right.
\end{aligned}
$$

$$
I_{\zeta}^{(4)}=\int_{-d}^{0} \frac{Z_{\zeta}(z)}{h} z \mathrm{~d} z=\left\{\begin{array}{l}
\frac{N_{0}^{-0.5}}{k_{0}^{2} h}\left\{k_{0} d \sinh \left[k_{0}(h-d)\right]-\cosh \left(k_{0} h\right)+\cosh \left[k_{0}(h-d)\right]\right\}, \quad \varsigma=0 \\
\frac{N_{\zeta}^{-0.5}}{k_{\varsigma}^{2} h}\left\{k_{\varsigma} d \sin \left[k_{\varsigma}(h-d)\right]+\cos \left(k_{\varsigma} h\right)-\cos \left[k_{\varsigma}(h-d)\right]\right\}, \quad \varsigma=1,2,3, \ldots
\end{array}\right.
$$

To evaluate the unknown coefficients $A_{m, l}^{(i)}$ and $B_{m, l}^{(i)}$, we take $(2 M+1)$ terms $(m=-M, \ldots, 0, \ldots, M)$ and $(L+1)$ terms $(l=0,1, \ldots, L)$ in Eqs. $(11)$ and $(14)$ and take $(\tau=-M, \ldots, 0, \ldots$, $M)$ and $(\zeta=0,1, \ldots, L)$ in Eqs. (A1) and (A6) as well, thus a $2(2 M+1)(L+1)$ order complex linear equation matrix is obtained, which can be used to calculate the same number of unknown coefficients $A_{m, l}^{(i)}$ and $B_{m, l}^{(i)}$. In all the semi-analytical computations as given in this paper, $M=12$ and $L=8$ are taken to lead to accurate results.

Appendix. B Proof process of $F_{\mathrm{R}, 1}^{(1)}=F_{\mathrm{R}, 2}^{(2)}, \quad F_{\mathrm{R}, 4}^{(4)}=F_{\mathrm{R}, 5}^{(5)}, F_{\mathrm{R}, 5}^{(1)}=-F_{\mathrm{R}, 4}^{(2)}$ for the case studied in Section 5.1

To make it easy understood, a sketch is given in Fig.B.1, in which $F_{\mathrm{R}}^{\prime}$ is the horizontal radiation force due to unit velocity amplitude of the structure oscillating in the direction $\theta=2 \pi / 3$. Apparently, due to symmetry property of the vertical cylinder with radius function $R(\theta)=0.5 h[1+0.1 \cos (3 \theta)]$, the horizontal radiation forces $F_{\mathrm{R}, 1}^{(1)}$ and $F_{\mathrm{R}}^{\prime}$ are in the same directions of the corresponding oscillating velocities, separately, and are also proportional to them in complex amplitude with the same scale factor $F_{\mathrm{R}, 1}^{(1)}$.

For the structure oscillating in any direction with unit velocity amplitude, the velocity amplitude denoted as $\mathrm{e}^{\mathrm{i} \theta}$ (as given in Fig.B.1), can be expressed by the sum of velocities 1 and $\mathrm{e}^{2 \mathrm{i} \pi / 3}$ as:

$$
\mathrm{e}^{\mathrm{i} \theta}=a_{1}+a_{2} \mathrm{e}^{2 \mathrm{i} \pi / 3}
$$

where $a_{1}$ and $a_{2}$ are two real parameters that can be easily determined.

The radiation force $F_{\mathrm{R}}^{\prime}$ corresponding to the velocity $\mathrm{e}^{2 \mathrm{i} \pi / 3}$ can be written as: 


$$
F_{\mathrm{R}}^{\prime}=F_{\mathrm{R}, 1}^{(1)} \mathrm{e}^{2 \mathrm{i} \pi / 3} .
$$

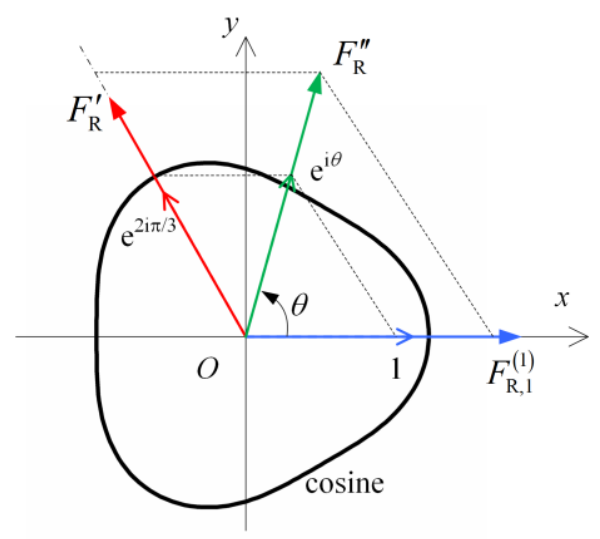

Fig. B.1 A sketch of radiation forces

Then the radiation force corresponding to $\mathrm{e}^{\mathrm{i} \theta}$, denoted as $F_{\mathrm{R}}^{\prime \prime}$, can be written as the sum of those due to $a_{1}$ and $a_{2} \mathrm{e}^{2 \mathrm{i} \pi / 3}$ as follows:

$$
F_{\mathrm{R}}^{\prime \prime}=a_{1} F_{\mathrm{R}, 1}^{(1)}+a_{2} F_{\mathrm{R}}^{\prime}=F_{\mathrm{R}, 1}^{(1)} \mathrm{e}^{\mathrm{i} \theta} .
$$

It means that the horizontal radiation force always equals to the product of $F_{\mathrm{R}, 1}^{(1)}$ and the unit velocity amplitude, regardless of the oscillating direction. For $\theta=\pi / 2, \quad F_{\mathrm{R}}^{\prime \prime}=F_{\mathrm{R}, 2}^{(2)} \mathrm{e}^{\mathrm{i} \pi / 2}=F_{\mathrm{R}, 1}^{(1)} \mathrm{e}^{\mathrm{i} \pi / 2}$, i.e. $F_{\mathrm{R}, 1}^{(1)}=F_{\mathrm{R}, 2}^{(2)}$.

Similarly, $F_{\mathrm{R}, 4}^{(4)}=F_{\mathrm{R}, 5}^{(5)}$ and $F_{\mathrm{R}, 5}^{(1)}=-F_{\mathrm{R}, 4}^{(2)}$ can also be proved, in which the minus sign is induced by the oppsite relationship between ( $n_{4}$ vs. $\left.n_{y}, y n_{z}\right)$ and $\left(n_{5}\right.$ vs. $\left.n_{x}, x n_{z}\right)$.

\section{References}

Bhatta, D.D., 2007. Computation of added mass and damping coefficients due to a heaving cylinder. J. Appl. Math. \& Computing. 23(1-2), 127-140.

Bhatta, D.D., Rahman, M., 2003. On scattering and radiation problem for a cylinder in water of finite depth. Int. J. Eng. Sci. 41, 931-967.

Chakrabarti, S.K., Chakrabarti, P., Krishna, M.S., 2006. Design, construction and installation of a floating caisson used as a bridge pier. Journal of Waterway, Port, Coastal and Ocean Engineering. 132(3), 143-156.

Chatjigeorgiou, I.K., 2013. The hydrodynamics of arrays of truncated elliptical cylinders. European Journal of Mechanics-B/Fluids. 37, 153-164.

Chatjigeorgiou, I.K., Mavrakos, S.A., 2010. An analytical approach for the solution of the hydrodynamic diffraction by arrays of elliptical cylinders. Applied Ocean Research. 32(2), 242-251.

Disibüyük, N.B., Korobkin, A.A., Yilmaz, O., 2017. Linear wave interaction with a vertical cylinder of arbitrary cross section: an asymptotic approach. Journal of Waterway, Port, Coastal, and Ocean 


\section{Engineering. DOI: 10.1061/(ASCE)WW.1943-5460.0000407}

Drobyshevski, Y., 2004. Hydrodynamic coefficients of a floating, truncated vertical cylinder in shallow water. Ocean Engineering. 31, 269-304.

Eidem, M.E., 2017. Overview of floating bridge projects in Norway. Proceedings of the ASME 2017 36th International Conference on Ocean, Offshore and Arctic Engineering. June 25-30, 2017. Trondheim, Norway. pp. 1-6.

Falnes, J., 2002. Ocean Waves and Oscillating Systems. Cambridge University Press.

Jiang, S.C., Gou, Y., Teng, B., 2013. Water wave radiation problem by a submerged cylinder. Journal of Engineering Mechanics. p.06014003. http://dx.doi.org/10.1061/(ASCE)EM.1943-7889.0000723.

Linton, C.M., Evans, D.V., 1992. The radiation and scattering of surface waves by a vertical circular cylinder in a channel. Philosophical Transactions of the Royal Society A. 338(1650), 325-357.

Liu, J., Guo, A., Fang, Q., et al., 2017. Investigation of linear wave action around a truncated cylinder with non-circular cross section. Journal of Marine Science and Technology. DOI: 10.1007/s00773-017-0516-0

Liu, J., Guo, A., Li, H., 2016. Analytical solution for the linear wave diffraction by a uniform vertical cylinder with an arbitrary smooth cross-section. Ocean Engineering, 126:163-175.

Sudhakar, S., Nallayarasu, S., 2011. Influence of heave plate on hydrodynamic response of Spar. Proceedings of the ASME 2011 30th International Conference on Ocean, Offshore and Arctic Engineering. June 19-24, 2011. Rotterdam, The Netherlands. pp. 1-11.

Wan, L., Magee, A.R., Hellan, , et al., 2017. Initial design of a double curved floating bridge and global hydrodynamic responses under environmental conditions. Proceedings of the ASME 2017 36th International Conference on Ocean, Offshore and Arctic Engineering. June 25-30, 2017. Trondheim, Norway. pp. 1-10.

Williams, A.N., Darwiche, M.K., 1988. Threee-dimensional wave scattering by elliptical breakwaters. Ocean Engineering. 15(2), 103-118.

Williams, A.N., Darwiche, M.K., 1990. Wave radiation by truncated elliptical cylinder. Journal of Waterway, Port, Coastal, and Ocean Engineering. 116(1), 101-119.

Yeung, R.W., 1981. Added mass and damping of a vertical cylinder in finite-depth waters. Applied Ocean Research, 3(3), 119-133.

Zhang, S., Williams, A.N., 1996. Wave radiation by a submerged elliptical disk. International Journal of Offshore and Polar Engineering, 6(1996), 35-40.

Zheng, S., Zhang, Y., 2015. Wave diffraction from a truncated cylinder in front of a vertical wall. Ocean Engineering. 104, 329-343.

Zheng, S., Zhang, Y., 2016. Wave radiation from a truncated cylinder in front of a vertical wall. Ocean Engineering. 111, 602-614.

Zheng, S., Zhang, Y., 2018. Theoretical modelling of a new hybrid wave energy converter in regular waves. Renewable Energy. 128, 125-141. 